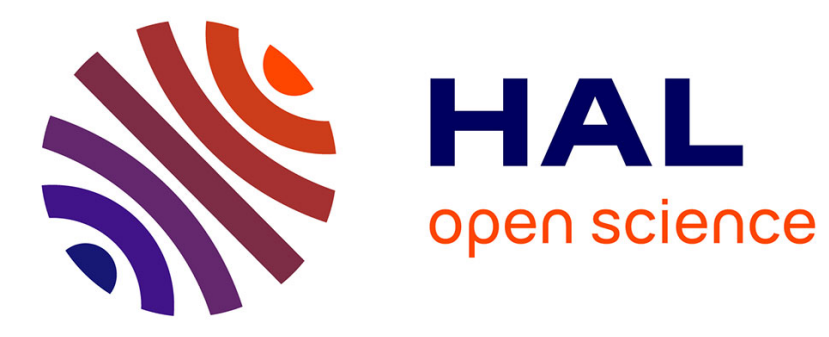

\title{
Turbidity data obtained from image analysis in near critical hydrogen
}

Ana Oprisan, Gurunath Gandikota, Denis Chatain, Yves Garrabos, Daniel Beysens

\section{- To cite this version:}

Ana Oprisan, Gurunath Gandikota, Denis Chatain, Yves Garrabos, Daniel Beysens. Turbidity data obtained from image analysis in near critical hydrogen. Physical Review E , 2019, 100 (5), 052112 (15 p.). 10.1103/PhysRevE.100.052112 . hal-02368476

\section{HAL Id: hal-02368476 https://hal.science/hal-02368476}

Submitted on 28 Jan 2021

HAL is a multi-disciplinary open access archive for the deposit and dissemination of scientific research documents, whether they are published or not. The documents may come from teaching and research institutions in France or abroad, or from public or private research centers.
L'archive ouverte pluridisciplinaire HAL, est destinée au dépôt et à la diffusion de documents scientifiques de niveau recherche, publiés ou non, émanant des établissements d'enseignement et de recherche français ou étrangers, des laboratoires publics ou privés. 


\title{
Turbidity data obtained from image analysis in near critical hydrogen
}

\author{
Ana Oprisan ๑* \\ Department of Physics and Astronomy, College of Charleston, 66 George Street, Charleston, South Carolina 29424, USA \\ Gurunath Gandikota and Denis Chatain \\ Université Grenoble Alpes and DSBT, IRIG, CEA, 38000 Grenoble, France \\ Yves Garrabos \\ ICMCB, UMR No. 5026, CNRS, Université de Bordeaux, and Bordeaux INP, 33600 Pessac, France \\ Daniel Beysens \\ Physique et Mécanique des Milieux Hétérogènes, CNRS, ESPCI, PSL Research University, and Sorbonne Université, \\ Sorbonne Paris Cité, Universités Paris 6 \& Paris 7, 10 rue Vauquelin, 75005 Paris, France \\ and Université Grenoble Alpes and DSBT, IRIG, CEA, 38000 Grenoble, France
}

(Received 13 June 2019; published 13 November 2019)

\begin{abstract}
Video images are being used with increased frequency in science, supplanting current methods such as light scattering by statistical evaluation of the images. In this study we use light turbidity data due to density-induced refractive index fluctuations to obtain critical amplitudes from image analysis. In order to bring hydrogen $\left(\mathrm{H}_{2}\right)$ very close to its critical point, we place the sample cell under weightlessness using a magnetic levitation device. Images of an $\mathrm{H}_{2}$-filled cell are taken near its critical temperature of $33 \mathrm{~K}$ by illuminating the cell with three different filters. We fit the turbidity data to a theoretical expression that allows us to estimate the critical amplitudes of isothermal compressibility and fluctuation correlation length. The values of isothermal compressibility and correlation length obtained from turbidity fitting are compared against literature values. Our data analysis shows a large sensitivity of the fitting parameters to the refractive index value and to even minute density deviations from criticality.
\end{abstract}

DOI: 10.1103/PhysRevE.100.052112

\section{INTRODUCTION}

We celebrate 150 years of critical phenomena studies that started with carbon dioxide liquefaction by Andrews. In his seminal work [1], Andrews introduced the concept of the critical point. It was van der Waals who introduced the principle of corresponding states, which asserts that the functional relations among pressure, temperature, and volume are the same for all fluids [2]. Among other optical techniques used for investigating critical phenomena, turbidity measures the amount of light transmitted through a fluid and helps probe the dynamics of density fluctuations near a fluid's critical point [3].

Close to the critical point, the physical properties of a fluid follow well-known universal laws [4-10]. When the critical point is approached, some parameters, such as the thermal expansion coefficient, isothermal compressibility, and thermal conductivity, diverge. Others, such as surface tension, liquidvapor density difference, capillary length, and coefficient of thermal diffusivity, vanish. The fact that the above parameters asymptotically approach zero when approaching the critical point means that gravity effects become very large, which requires weightlessness for studying critical point phenomena. By heating or cooling a fluid, one can approach or move

\footnotetext{
*oprisana@cofc.edu
}

away from the liquid-vapor critical point. As a result, one can modify the properties of the liquid and vapor phases in a scaled way and thus study the liquid-vapor interface for a variety of fluid conditions.

Hydrogen $\left(\mathrm{H}_{2}\right)$ is a key player in space technology for propulsion and cooling $[11,12]$. Fluid management under the significant acceleration during takeoff and under microgravity in outer space poses significant technological challenges to space missions [13]. Among other difficulties, effective fluid management in microgravity requires a better understanding of thermo-hydrodynamics and phase change once buoyancy forces are canceled. Performing classical microgravity experiments with hydrogen is nearly impossible due to safety concerns [14], which leaves us with the alternative of counterbalancing gravity by using the diamagnetic property of $\mathrm{H}_{2}$ [15].

We measured the turbidity dependence on temperature $T$ for a fluid $\mathrm{H}_{2}$ cell near its critical temperature $T_{c}$ using three different optical filters, which allowed turbidity estimation at different wavelengths. In this study, we used the hydrogen levitation device (HYLDE) that allows gravity compensation by volumic magnetic forces induced by a magnetic field gradient (see $[16,17]$ for an introduction to magnetic compensation of gravity and $[14,15,18]$ and references therein for the mathematical derivation of effective gravity under magnetic compensation). 
Video images are increasingly used in many areas of science and technology as they allow a better understanding of physical processes than the more traditional light scattering methods. We show that image analysis of turbidity gives the same information on compressibility and correlation length of $\mathrm{H}_{2}$ critical fluid as one gets with classical light scattering. By fitting the turbidity $\tau$ versus reduced temperature $(\epsilon=$ $\left.T / T_{c}-1\right)$ with well-established theoretical formulas [19-23] we found the values of two critical amplitudes: $\kappa_{T 0}$ for the isothermal compressibility and $\xi_{0}$ for the fluctuation correlation length.

Among the benefits of our approach, we mention that magnetic compensation of gravity allows a very close approach to critical temperature without gravity disturbances that usually affect critical fluids, e.g., buoyancy-driven instabilities and strong density gradients. The HYLDE cryogenics system uses a low-power, adjustable, light source to minimize the temperature disturbance of the probe. Another advantage of our approach is that we can visualize the whole sample, which allows us to detect density inhomogeneities. Working with three different filters centered on red, green, and blue wavelengths allows us to cross-check estimated values for susceptibilities based on turbidity measurements. Finally, we can use the same setup to study other critical phenomena, such as phase transition, and determine critical temperature with high precision (outside the scope of this study).

The present paper is organized as follows. First, we briefly review background information on both critical point scaling laws and turbidity. Next, the HYLDE setup is reviewed without repeating all the details of previous studies. Our results start with a critical review of existing data on $\mathrm{H}_{2}$ critical parameters and the refractive index measurements near the critical point. The bulk of the section on results is concerned with extracting the critical amplitudes $\kappa_{T 0}$ and $\xi_{0}$ by fitting the turbidity $\tau$ versus reduced temperature $\epsilon$ graphs. We also compare our results against existing experimental data and theoretical predictions. Finally, we provide insight into the expected power law exponents predicted by the existing theoretical model of turbidity via numerical simulations.

\section{BACKGROUND}

\section{A. Critical point phenomena}

Near the liquid-vapor critical point, a number of thermodynamic quantities diverge asymptotically as power laws when temperature is varied $[4,5,9,24]$. Among others, the isothermal compressibility $\kappa_{T}$ and the correlation length of density fluctuations $\xi$ both diverge as power laws near the critical temperature $T_{c}$. For example, the susceptibility $\chi$ and the fluctuation correlation length $\xi$ are written as [4]

$$
\begin{aligned}
& \chi=p_{c} \kappa_{T}=\Gamma_{0} \epsilon^{-\gamma}\left(1+a_{\chi} \epsilon^{\Delta}+\cdots\right), \\
& \xi=\xi_{0} \epsilon^{-\nu}\left(1+a_{\xi} \epsilon^{\Delta}+\cdots\right) .
\end{aligned}
$$

Here $p_{c}$ is the critical pressure and $\epsilon=\frac{T}{T_{c}}-1$ is the reduced temperature, with $T$ and $T_{c}$ the temperature and critical temperature, respectively. In addition, $\Gamma_{0}=p_{c} \kappa_{T 0}$ and $\xi_{0}$ are critical amplitudes that depend on the considered fluid; $\gamma=1.24$ and $v=0.63$ are universal critical exponents. The existence of universal relations, the so-called scaling laws, between exponents means that only two critical exponents are independent. Consequently, all the amplitudes are connected by universal ratios and only two critical amplitudes are independent $[8-10,25]$. Therefore, the experimental estimation of the amplitudes, such as $\Gamma_{0}$ and $\xi_{0}$ from Eq. (1), is of prime importance for the validation of the two-scale-factor universality for simple fluids that are expected to belong to the three-dimensional Ising-like systems [8,10,25]. In Eq. (1) we also note that the terms inside the parentheses correspond to nonanalytical corrections characterized by the universal value $\Delta=0.50$ of the corresponding exponent $[26,27]$. The amplitudes of the nonanalytical correction such as $a_{\chi}$ or $a_{\xi}$ are fluid dependent and only one among these nonanalytic amplitudes can be used to characterize each fluid (see, for example, [28]). Typically, their magnitudes are of the order of unity. Since in this study the temperature remains close to $T_{c}$, the small contributions of nonanalytical amplitudes are not taken into account hereafter. As a result, we are only concerned with the estimation of critical amplitudes $\Gamma_{0}$ and $\xi_{0}$ from turbidity observed in recorded video images of a critical $\mathrm{H}_{2}$ cell.

\section{B. Turbidity}

Turbidity is obtained from measured light transmission $\mathscr{T}$ through a sample with thickness $e$ [19-21],

$$
\mathscr{T}=I_{T} / I_{0},
$$

where $I_{T}$ is the transmitted light intensity and $I_{0}$ is the incident light intensity. Turbidity can be calculated as

$$
\tau=-\frac{1}{e} \ln (\mathscr{T})
$$

Very close to critical point, turbidity is only due to the Rayleigh scattering of light [19]. The enhancement of refractive index fluctuations due to the density (order parameter) fluctuations becomes increasingly important as one approaches the critical point and leads to the so-called critical opalescence phenomenon [4-6,9]. According to the OrnsteinZernike theory, turbidity is related to the scattered light intensity integrated over all scattered angles [22,23]

$$
I(k)=A T \kappa_{T} \sin ^{2}(\Phi) /\left[1+(q \xi)^{2}\right],
$$

where $q$ represents the amplitude of the transfer wave vector between the incident wave vector $k_{i}$ and scattered $k_{s}$, i.e., $q=$ $\left|k_{i}-k_{s}\right|=2 k_{0} \sin (\theta / 2)$. The wave vector of the incident light is $k_{0}=2 \pi n / \lambda_{0}, \Phi$ is the angle between the polarization of the incident beam wave vector and the scattered light wave vector, $n$ is the refractive index of the fluid, $\lambda_{0}$ is the wavelength of the incident light in the vacuum, and $\theta$ is the scattering angle. The factor $A$ is given by [19-21]

$$
A=\frac{\pi^{2}}{\lambda_{0}^{4}}\left(\rho \frac{\partial n^{2}}{\partial \rho}\right)_{T}^{2} k_{B}=\frac{\pi^{2}}{\lambda_{0}^{4}}\left(\frac{\left(n^{2}-1\right)\left(n^{2}+2\right)}{3}\right)^{2} k_{B},
$$

where $k_{B}$ is the Boltzmann constant. The factor $A$ varies strongly with both $\lambda_{0}$ (due to the $\lambda_{0}^{-4}$ factor) and with the 
index of refraction $n$. The index of refraction $n$ corresponds to $\lambda_{0}$ at $T=T_{c}$ and $\rho=\rho_{c}$. For the derivative $\frac{\partial n^{2}}{\partial \rho}$, we used Lorentz-Lorenz relationship

$$
\frac{1}{\rho} \frac{n^{2}-1}{n^{2}+2}=r(\lambda)
$$

where $r(\lambda)$ is almost constant at a given wavelength.

It should be noticed that varying the wavelength from blue $(0.45 \mu \mathrm{m})$ to red $(0.65 \mu \mathrm{m})$ has the same effect on turbidity as varying the sample's thickness by a factor

$$
\frac{e_{1}}{e_{2}}=\frac{\ln \left(\lambda_{1}\right)}{\ln \left(\lambda_{2}\right)} \approx 2 .
$$

Indeed, consider two samples of thicknesses $e_{1}$ and $e_{2}$, illuminated by light with wavelengths $\lambda_{1}$ and $\lambda_{2}$, respectively. From Eq. (3) and the definition of the factor $A$ in Eq. (5) one gets, for the ratio of turbidities of the two samples,

$$
\frac{\tau_{2}}{\tau_{1}}=\frac{e_{1}}{e_{2}} \frac{\ln \left(\lambda_{2}\right)}{\ln \left(\lambda_{1}\right)},
$$

which leads to Eq. (7) for $\tau_{1}=\tau_{2}$.

Integrating Eq. (4) over all scattering angles, one gets the turbidity [19-21]

$$
\tau=A \pi T_{c}(1+\epsilon) \kappa_{T} F(a)+\tau_{B} .
$$

The parameter $\tau_{B}$ is a background contribution due to the light attenuation other than scattering by critical fluctuations, e.g., Brillouin and Raman scattering and light reflection or absorption by the windows. The background turbidity $\tau_{B}$ also includes the effect of uncertainty in the estimation of $I_{0}$. The function $F(a)$ is given by [19-21]

$$
F(a)=\left(\frac{2 a^{2}+2 a+1}{a^{3}}\right) \ln (1+2 a)-2\left(\frac{1+a}{a^{2}}\right)
$$

and represents an interference term which depends on $a=$ $2\left(k_{0} \xi\right)^{2}$ and whose limit value for $\epsilon \rightarrow \infty$ is 8/3 [29]. For a reasonable range of $1 \AA \leqslant \xi_{0} \leqslant 3 \AA$ and all wavelengths in the visible spectrum, the interference function $F(a)$ in Eq. (9) is at least at $77.7 \%$ of its limit value of $8 / 3$ for all $\epsilon>10^{-3}$. In other words, for $\epsilon>10^{-3}$ the interference function is quasiconstant and its effect on turbidity formula given by Eq. (8) can be neglected. This approximation corresponds in $\mathrm{H}_{2}$ to an off-critical temperature of about $T-T_{c}>33 \mathrm{mK}$ (see the discussions on Fig. 5 in Sec. IV C below regarding the asymptotic behavior of turbidity for both large and very small values of reduced temperature).

\section{EXPERIMENTS AND METHODS}

\section{A. Magnetic levitation setup}

Due to earth's gravity, fluids near the critical point compress under their own weight. Additionally, due to the divergence of the thermal expansion coefficient near the critical point, minute temperature gradients induce strong convective flows inside critical fluids $[4-7,9,10,30]$. In order to compensate for these strong gravity effects, we carried out the experiments using the HYLDE cryogenic facility [14-16]. The
HYLDE setup uses a magnetic field up to $10 \mathrm{~T}$ generated by a cylindrical superconducting coil. Hydrogen is diamagnetic; thus it can levitate near the upper end of the coil where a nearly constant magnetogravitational potential field is produced. Gravitational field compensation is possible because a diamagnetic substance, such as $\mathrm{H}_{2}$, placed in a magnetic field is subject to a force that is proportional to its magnetic susceptibility and to the local gradient $(\vec{B} \cdot \vec{\nabla}) \vec{B}$ [see Eq. (1) in [14] and Eq. (1) in [16]]. The theoretical background for magnetic compensation of gravity in $\mathrm{H}_{2}$ is detailed in [16]. With respect to the experimental setup, without repeating the very detailed description and schematics presented in [16], we only mention that the apparatus consists of a cryostat that contains the superconducting coil and an anticryostat containing the sample and the optics [see Fig. 1 in [16], Fig. 3(a) in [18], and Fig. 1(a) in [14] for details]. The Nb-Ti superconducting coil inside the cryostat is dipped in liquid helium at a temperature of $2.16 \mathrm{~K}$ and a pressure of $0.1 \mathrm{MPa}$. The experimental cell is mounted inside the anticryostat and maintained under a vacuum of less than $10^{-7}$ mbar. Endoscopes for a light source and a video camera are mounted inside the anticryostat. The positions of the endoscopes can be adjusted independently. A more detailed schematics of HYLDE is shown in Fig. 1(a) of [31] or [14] together with an actual photo of the experimental cell of size $7 \times 7 \times 7 \mathrm{~mm}^{3}$ [see Fig. 1(b) in [31] or [14]]. The same HYLDE setup was used in [32] (see their Fig. 10) with a $3 \times 3 \times 3 \mathrm{~mm}^{3}$ cell.

It has been demonstrated [17] that the magnetic levitation technique using a solenoid of cylindrical configuration only achieves total compensation of gravity at a single point in space. As a result, there is always a residual gravitational field radially directed towards the center of the cell. The uniformity of the gravitational field depends on the size of the cell (see the in-depth analysis of the effective gravity homogeneity inside cylindrical samples under magnetic levitation in [16]). Using the HYLDE setup, a uniform magnetogravitational potential field better than $1 \%$ and $2.5 \%$ can be achieved in cells of side $h=3$ and $7 \mathrm{~mm}$, respectively [16].

Hydrogen can be found in two states of spin polarization: ortho and para. When $\mathrm{H}_{2}$ is cooled down, the balance between ortho- $\mathrm{H}_{2}\left(o-\mathrm{H}_{2}\right)$ and para- $\mathrm{H}_{2}\left(p-\mathrm{H}_{2}\right)$ is shifted and the percentage of $p-\mathrm{H}_{2}$ (antiparallel spins) increases from about $33 \%$ at room temperature to $96 \%$ at $30 \mathrm{~K}$ (see [33]). As the temperature decreases towards $T_{c}$, more $o-\mathrm{H}_{2}$ (parallel spins of $\mathrm{H}$ nuclei) transforms into $p-\mathrm{H}_{2}$, which in turn determines a decrease of $T_{c}$ itself. In our experiment, the change in temperature was much faster than the slow drift of $T_{c}$ because of $o-\mathrm{H}_{2}$ to $p-\mathrm{H}_{2}$ conversion. It has been found that $T_{c}$ follows an exponential relaxation with a time constant of about $2500 \mathrm{~min}$ [34]. As the experiments are performed within hours after filling the cell with the fluid initially at room temperature, the $\mathrm{H}_{2}$ state is thus normal- $\mathrm{H}_{2}\left(n-\mathrm{H}_{2}\right)$. Its critical point is defined by a range of values for $T_{c}, p_{c}$, and $\rho_{c}$ given in Tables I and II.

Hydrogen is filled inside the cell by using a capillary tube of inner diameter $0.5 \mathrm{~mm}$. The capillary tube is fitted with a thermal switch made of a small block of copper continuously cooled under the triple point of $\mathrm{H}_{2}$ by a copper wire connected to the helium bath of the cryostat. The switch is heated using a resistive heater. In the absence of heating, the $\mathrm{H}_{2}$ inside 
TABLE I. Relevant critical parameters for $n-\mathrm{H}_{2}$ based on our extensive review of the existing literature.

\begin{tabular}{lcrr}
\hline \hline$T_{c}(\mathrm{~K})$ & $p_{c}$ & $\rho_{c}$ & Polarizability $\left(\mathrm{cm}^{3} / \mathrm{mol}\right)$ \\
\hline 33.18 & $9864.8 \mathrm{mmHg}$ & $29.87 \mathrm{~kg} / \mathrm{m}^{3}$ & Ref. \\
& & $29.88 \mathrm{~kg} / \mathrm{m}^{3}$ & 2.0372 \\
33.19 & $9864.8 \mathrm{mmHg}$ & $30.12 \mathrm{~kg} / \mathrm{m}^{3}$ & {$[35]$} \\
33.19 & $9865 \mathrm{mmHg}$ & $14.94 \times 10^{-3} \mathrm{~mol} / \mathrm{cm}^{3}$ & {$[36]$} \\
33.19 & $1.315 \mathrm{MPa}=12.98 \mathrm{~atm}$ & $30.12 \mathrm{~kg} / \mathrm{m}^{3}=14.94 \mathrm{~mol} / 1$ & 2.0372 \\
33.18 & $12.98 \mathrm{~atm}$ & $30.12 \mathrm{~kg} / \mathrm{m}^{3}=0.01494 \mathrm{~mol} / \mathrm{cm}^{3} 1$ & 2.0372 \\
& & & {$[38]$} \\
& & & {$[39]$} \\
\hline \hline
\end{tabular}

the capillary tube freezes, thus closing the cell. To fill or empty the cell, the switch is heated up, thus melting solid $\mathrm{H}_{2}$ inside the capillary tube. To determine the critical density, the cell is filled up to half its height at a temperature to within $50 \mathrm{mK}$ of $T_{c}$ and the meniscus is monitored under $g=1$ while temperature slowly increases from $T_{c}-50 \mathrm{mK}$ towards $T_{c}$. If the level of the meniscus does not change within the optical resolution while changing the temperature, it means that the cell is filled at its critical density (see also [34] for a detailed description of the procedure). A measuring accuracy of $0.2 \mathrm{~mm}$ for the meniscus corresponds to a density uncertainty of $\left(\rho-\rho_{c}\right) / \rho_{c} \approx 2 \%$. The experimental cell is provided with thermal bridges, strands of copper wires connecting the bottom flange of the anticryostat, which is maintained at liquid helium temperature. Resistive heaters in thermal contact with the cell are used for both heating and controlling the temperature of the cell. The temperature is monitored by two thermometers attached on the cell's wall. The temperature control of the cell achieved with a standard proportional-integral-derivative control system is better than $0.3 \mathrm{mK}$.

\section{B. Turbidity measurement method}

Traditionally, light transmission is measured with a thin $(0.1 \mathrm{~mm})$ polarized laser beam. Here light transmission is measured by a fixed gain camera on the image itself, pixel by pixel or averaged over a window (Fig. 1). In order to avoid collecting multiple scattered light, the total aperture angle of the collecting lens is small $\left(\alpha= \pm 0.75^{\circ}\right.$ or $\left.\alpha=13 \mathrm{mrad}\right)$, similar to laser transmission methods. This small aperture is the result of a long depth ( $0.4 \mathrm{~m}$ typically) of focus. The CCD camera resolution was $1024 \times 1024$ pixels at 30 frames/s [34], although because of the small aperture of the optical system only about $750 \times 750$ pixels were used. Incident light is produced by a collimated beam originating from a whitelight lamp with switchable interferential filters centered on blue $(465.2 \mathrm{~nm}$, bandwidth $71 \mathrm{~nm})$, green $(519.4 \mathrm{~nm}$, bandwidth $92 \mathrm{~nm})$, and red (669.4 nm, bandwidth $78 \mathrm{~nm})$.

A detailed view of the cell is shown in Fig. 2 (see also Fig. 2 in [34] for more details on the optical parameters of the HYLDE). The cell is cylindrical with a sapphire window at one end and a mirror at the other end [see Fig. 2(a)]. Its thickness is $e_{0}=21.00 \mathrm{~mm}$ and its radius is $20 \mathrm{~mm}$. The mirror placed at the end of the cell reflects the light back, which makes the final light path through the fluid $e=2 e_{0}$. Compared to a laser beam, which typically heats up the fluid by $1 \mathrm{mK} / \mathrm{mW}$, this method has the advantage of dissipating less power.

We used an eight-bit CCD camera, which allowed for 256 gray-level discretization of recorded images. In gray levels, the maximum intensity is 255 [see Figs. 3(a) and 3(b)] and the minimum we recorded is about 6 [see Figs. 3(c) and 3(d)], which corresponds to the electronic noise of the camera. As a result, the minimum detectable transmission with our setup is $\mathscr{T}_{\min } \approx 6 / 255 \approx 0.0235$, which corresponds to a maximum turbidity $\tau_{\max }=(-1 / e) \ln \left(\mathscr{T}_{\max }\right) \approx 100 \mathrm{~m}^{-1}$. Accordingly, the maximum transmission is $\mathscr{T} \approx(255-6) / 255 \approx$ 0.976 , which corresponds to a minimum turbidity value of $\tau_{\min }=(-1 / e) \ln \left(\mathscr{T}_{\min }\right) \approx 0.6 \mathrm{~m}^{-1}$. In Fig. 3 we show two representative images of the cell far from the critical point [see Figs. 3(a) and 3(b)] and closer to $T_{c}$ [see Figs. 3(c) and 3(d)]. The histograms of gray-level intensities [Figs. 3(c) and $3(d)]$ correspond to the squares marked and placed at the same location in the corresponding images [Figs. 3(a) and 3(b)]. As the temperature approaches $T_{c}$, the histogram becomes narrower, i.e., towards more homogeneous illumination, and shifts to lower light intensities.

TABLE II. Relevant critical parameters for $p-\mathrm{H}_{2}$ based on our extensive review of the existing literature.

\begin{tabular}{lcrr}
\hline \hline$T_{c}(\mathrm{~K})$ & $p_{c}$ & $\rho_{c}$ & Polarizability \\
\hline 32.976 & $9696.84 \mathrm{mmHg}$ & $31.43 \mathrm{~kg} / \mathrm{m}^{3}$ & Ref. \\
32.938 & $1.28377 \mathrm{MPa}$ & $15.556 \mathrm{~mol} / \mathrm{dm}^{3}$ & {$[37]$} \\
32.976 & $9696.8 \mathrm{mmHg}$ & $0.015 .59 \mathrm{~mol} / \mathrm{cm}^{3}$ & {$[41]$} \\
32.933 & & $0.03136 \mathrm{~g} / \mathrm{cm}^{3}$ & {$[35]$} \\
32.994 & $12.770 \mathrm{~atm}$ & $1 / 62.8 \mathrm{~mol} / \mathrm{cm}^{3}$ & {$[42]$} \\
32.976 & $1.2928 \mathrm{MPa}=12.759 \mathrm{~atm}$ & $31.43 \mathrm{~kg} / \mathrm{m}^{3}=15.59 \mathrm{~mol} / 1$ & {$[43]$} \\
32.976 & $12.759 \mathrm{~atm}$ & $0.01559 \mathrm{~mol} / \mathrm{cm}^{3}$ & $2.0279 \mathrm{~cm}^{3} / \mathrm{mol}^{3}$ \\
& & & {$[39]$} \\
\hline \hline
\end{tabular}




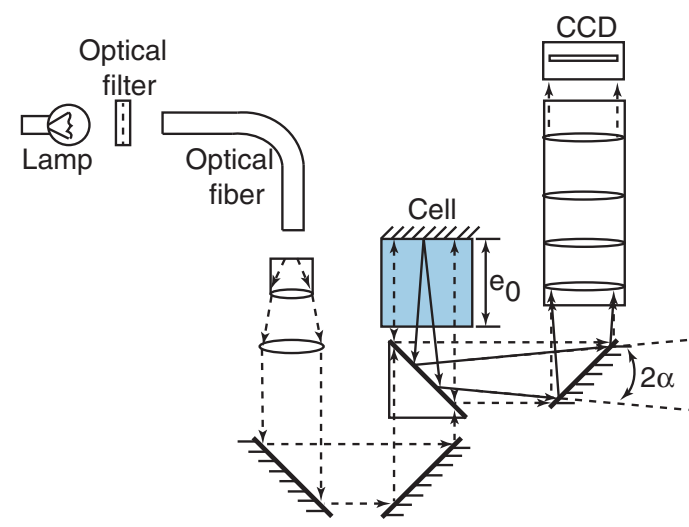

FIG. 1. Schematic representation of the HYLDE optical setup. The total angular aperture is $2 \alpha=26 \mathrm{mrad}$. The solid arrows indicate the $2 \alpha$ aperture.

\section{RESULTS}

\section{A. Critical review of the literature on critical parameters for hydrogen}

The turbidity given by Eq. (8) is sensitive to selected values for $n$ and $\lambda_{0}$. To assess its sensitivity, we performed a first-order (linear) perturbation of the logarithm of Eq. (8) and found

$$
\begin{aligned}
\frac{\delta \tau}{\tau}= & \left(\frac{1}{A} \frac{\partial A}{\partial n}+\frac{1}{F(a)} \frac{d F(a)}{d a} \frac{\partial a}{\partial n}\right) \delta n \\
& +\left(\frac{1}{A} \frac{\partial A}{\partial \lambda}+\frac{1}{F(a)} \frac{d F(a)}{d a} \frac{\partial a}{\partial \lambda}\right) \delta \lambda .
\end{aligned}
$$

The refractive index sensitivity is dominated by the first term $\frac{1}{A} \frac{\partial A}{\partial n}=\frac{4 n\left(1+2 n^{2}\right)}{\left(n^{2}-1\right)\left(n^{2}+2\right)}$. For $n=1.05$ this term $\frac{1}{A} \frac{\partial A}{\partial n} \approx 40$ and for $n=1.04$ the same term is $\frac{1}{A} \frac{\partial A}{\partial n} \approx 50$. The first factor

(a)

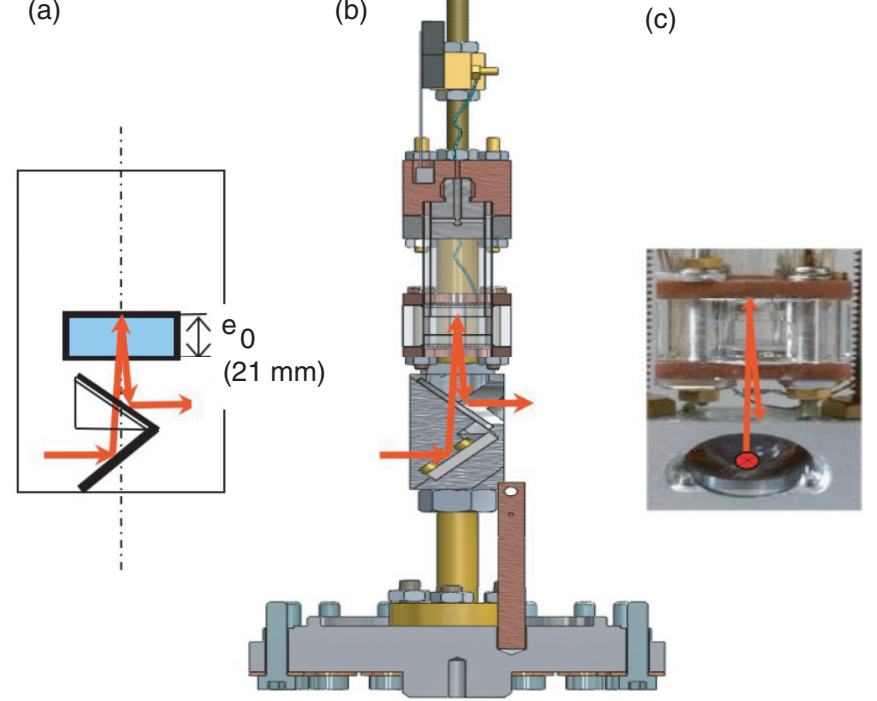

FIG. 2. Hydrogen sample cell and optical paths of $e=2 e_{0}=$ $2 \times 21 \mathrm{~mm}$ in the fluid. (a) Schematics. (b) Details. (c) Photo. (a)
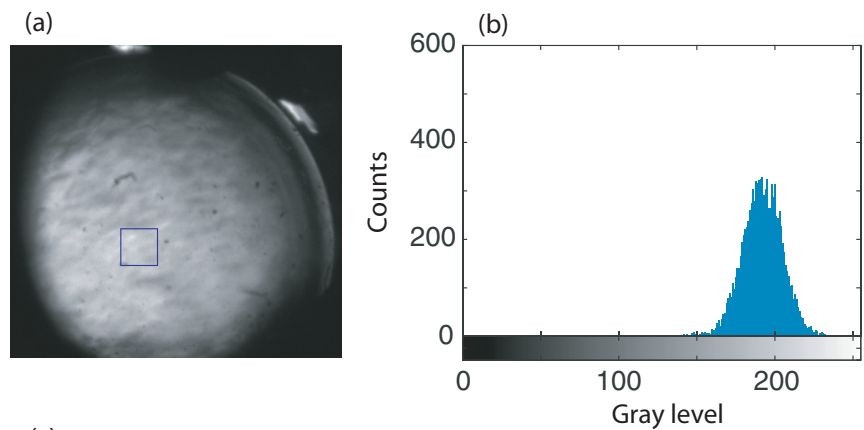

(c)
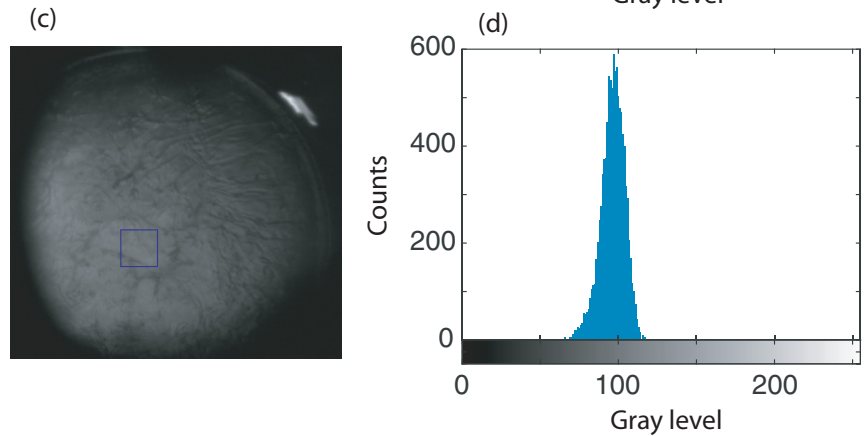

FIG. 3. Images of (a) and (c) the sample cell and (b) and (d) the corresponding gray-level histograms of the region of interest. Far from the critical point of $\mathrm{H}_{2}, T-T_{c}=993.5 \mathrm{mK}$, (a) the image is bright and (b) the gray-level distribution shifts towards lighter levels. Closer to critical point $T-T_{c}=2 \mathrm{mK}$, (c) there is less transmitted light and (d) the corresponding histogram shifts towards darker gray levels.

in the second term of $\delta n$ is $\frac{1}{F(a)} \frac{d F(a)}{d a} \rightarrow 1$ and the second factor $\frac{\partial a}{\partial n} \rightarrow 0$ as $T \rightarrow T_{c}$. Similarly, wavelength sensitivity is dominated by the first term $\frac{1}{A} \frac{\partial A}{\partial \lambda}=\frac{4}{\lambda}$, whereas the second term vanishes as $T \rightarrow T_{c}$. Therefore, the dominant terms, in absolute value, that drive the relative error of turbidity are

$$
\frac{\delta \tau}{\tau}=\frac{4 n^{2}\left(1+2 n^{2}\right)}{\left(n^{2}-1\right)\left(n^{2}+2\right)} \frac{\delta n}{n}+4 \frac{\delta \lambda}{\lambda} .
$$

Of the two terms above, the relative error on the refractive index $\delta n / n$ has a factor that is one order of magnitude larger than the wavelength uncertainty $\delta \lambda / \lambda$. This justifies our indepth review of the literature while searching for accurate refractive indices near $T_{c}$.

We thoroughly reviewed the literature for available data regarding $n-\mathrm{H}_{2}$ (see Table I) and $p-\mathrm{H}_{2}$ (see Table II). Our experiment was performed shortly after the $\mathrm{H}_{2}$ reached the critical temperature such that the liquid likely did not reach ortho-para equilibrium and $n-\mathrm{H}_{2}$ dominates in our experiment.

\section{B. Index of refraction}

The refractive index $n$ has been derived in two different ways: (i) from the Lorentz-Lorenz formula of specific refraction

$$
r=\left(n^{2}-1\right) /\left(n^{2}+2\right) / \rho
$$

or (ii) from the Clausius-Mossotti relation

$$
\mathscr{R}_{\mathrm{CM}}=(\phi-1) /(\phi+2) / \rho,
$$


TABLE III. Refraction index $n_{c}$ near critical temperature. The wavelengths in our experiment were $669.4 \mathrm{~nm}$ (red), $519.4 \mathrm{~nm}$ (green), and $465.2 \mathrm{~nm}$ (blue).

\begin{tabular}{|c|c|c|c|c|c|c|c|c|}
\hline \multicolumn{4}{|c|}{$n-\mathrm{H}_{2}$} & \multicolumn{4}{|c|}{$p-\mathrm{H}_{2}$} & \multirow[b]{2}{*}{ Source } \\
\hline$n_{c}$ & Red & Green & Blue & $n_{c}$ & Red & Green & Blue & \\
\hline \multirow{2}{*}{$1.04911(546.2 \mathrm{~nm})$} & & & & $1.04667-1.04706$ & & & & {$[38,44]$} \\
\hline & 1.04857 & 1.04929 & 1.04977 & & $1.04613-1.04652$ & $1.04685-1.04724$ & $1.04733-1.04772$ & {$[38,44] \&$ Eq. $(15)$} \\
\hline \multirow[t]{4}{*}{1.04726} & & & & $1.04489-1.04527$ & & & & {$[44]$} \\
\hline & 1.04857 & 1.04929 & 1.04977 & & $1.04613-1.04652$ & $1.04685-1.04724$ & $1.04733-1.04772$ & [44] \& Eq. (13) \\
\hline & & & & $1.04544-1.04582$ & & & & [41] \\
\hline & & & & 1.050958 & $1.04624-1.04663$ & $1.04679-1.04718$ & $1.04713-1.04753$ & $\begin{array}{c}\text { [41] \& Eq. (13) } \\
{[42]}\end{array}$ \\
\hline
\end{tabular}

where $\phi=n_{\infty}^{2}$ is the dielectric constant and $n_{\infty}$ is the refractive index for infinite wavelength. The value of $\mathscr{R}_{\mathrm{CM}}$ can be experimentally determined from the polarizability using $\mathscr{R}_{\mathrm{CM}}=P / M$, where $M$ is the molecular weight and $P$ is the molar polarizability. Once determined, the value of $\mathscr{R}_{\mathrm{CM}}$ is then used to estimate the dielectric constant $\phi$ and the corresponding refractive index $n_{\infty}$. To avoid notation confusion, we use $\phi$ for the dielectric constant throughout the paper and reserve $\epsilon$ only for the reduced temperature.

While reviewing the literature on the refractive index measurements near the critical point, we found two sets of data: (a) the index of refraction is given at a particular wavelength, or set of wavelengths, and (b) the wavelength is not specified (see Table III). The second case occurs because the Clausius-Mossotti relationship (13) was used to determine the refractive index $n_{\infty}$ from the dielectric constant. In both cases, we computed wavelength corrections for the refractive index based on Cauchy's formula of specific refraction [45]

$$
r(\lambda)=r_{\infty}+A / \lambda^{2}+B / \lambda^{4},
$$

with $A=0.7799569 \times 10^{6}$ and $B=0.495126 \times 10^{12}$ for $\lambda$ in angstroms and $r$ in $\mathrm{cm}^{3} / \mathrm{g}$. However, if the specific refraction is in $\mathrm{mol} / \mathrm{dm}^{3}$ as in [41], then Cauchy's coefficients must be multiplied by the molar mass/1000.

Based on Eq. (12), the refractive index is calculated with

$$
n(\lambda)=\sqrt{\frac{1+2 \rho r(\lambda)}{1-\rho r(\lambda)}} .
$$

Sometimes the specific refraction or the refraction index was measured using interferometry methods at a given wavelength. In such cases, when the wavelength $\lambda_{1}$ is known, to avoid uncertainties regarding $r_{\infty}$, we computed the refractive index at the wavelengths in our experiment $n\left(\lambda_{2}\right)$ based on a known value of the refractive index $n\left(\lambda_{1}\right)$. Based on Eqs. (12) and (14) one gets

$$
\frac{n_{1}^{2}-1}{n_{1}^{2}+2} \frac{1}{\rho}=r_{\infty}+A / \lambda_{1}^{2}+B / \lambda_{1}^{4}
$$

at a given wavelength $\lambda_{1}$. By rewriting the same relationship at a different wavelength $\lambda_{2}$ and eliminating $r_{\infty}$ one gets

$$
n\left(\lambda_{2}\right)=\sqrt{\frac{n\left(\lambda_{1}\right)^{2}+2 \beta}{1-\beta}},
$$

with $\beta=\rho \frac{n\left(\lambda_{1}\right)^{2}+2}{3}\left(\frac{1}{\lambda_{2}^{2}}-\frac{1}{\lambda_{1}^{2}}\right)\left[A+B\left(\frac{1}{\lambda_{2}^{2}}+\frac{1}{\lambda_{1}^{2}}\right)\right]$. Equation (17) has been used to handle the calculation of wavelengthdependent refractive indices when the refractive index is known at a specific wavelength $\lambda_{1}$ (see rows 1 and 2 in Table III).

\section{Refractive index values at $T_{c}$ (Table III)}

Souers derived a simple equation for all liquid and gases from 30 to $100 \mathrm{~K}[38,44]$,

$$
n(546.2 \mathrm{~nm})=1+3.15 \times 10^{-6} \rho,
$$

where the density $\rho$ is in $\mathrm{mol} / \mathrm{m}^{3}$, with molar weight of $\mathrm{H}_{2}$ equal to $2.01594 \mathrm{~g} / \mathrm{mol}$. It has been stated that the above equation "is a reasonable estimating procedure for all hydrogen isotopes in all phases. It will probably be accurate to a few percent" [44]. A crude estimate of the index of refraction with the above formula at $T_{c}$ gives (see the first row of Table III) $n_{c}=1.04667-1.04706$ for $p-\mathrm{H}_{2}$ and $n_{c}=$ 1.04911 for $n-\mathrm{H}_{2}$. Since we know the wavelength $\lambda_{1}$ for which the empirical formula (18) is valid, we used Cauchy's dispersion formula [45] (see the second rown in Table III) to correct the index of refraction value for our filters according to Eq. (17).

Stewart [44] derived a specific refraction formula from measurements of the dielectric constant. Stewart's formula is for $p-\mathrm{H}_{2}$ and is limited to temperatures below $100 \mathrm{~K}$ and densities below $0.080 \mathrm{~g} / \mathrm{cm}^{3}$. The density dependence of specific refraction (see p. 13 in [45]) is

$$
r_{\infty}=1 / \mathscr{P}=0.99575-0.09069 \rho+1.1227 \rho^{2},
$$

where polarizability $\mathscr{P}$ is in $\mathrm{cm}^{3} / \mathrm{g}$ and $\rho$ in $\mathrm{g} / \mathrm{cm}^{3}$. With $r_{\infty}$ from Eq. (19) we estimated the refractive index using Eq. (12) to be $1.04489 \leqslant n_{c} \leqslant 1.04527$ for $p-\mathrm{H}_{2}$ with densities in the range $29.87 \mathrm{~kg} / \mathrm{m}^{3} \leqslant \rho_{c} \leqslant 30.12 \mathrm{~kg} / \mathrm{m}^{3}$, and $n_{c}=1.04726$ for $n-\mathrm{H}_{2}$ with $\rho_{c}=31.43 \mathrm{~kg} / \mathrm{m}^{3}$. We also used Eq. (14) to account for Cauchy's correction (see row 4 in Table III).

Koch [46] determined the refractive index at 20 wavelengths from 230.2 to $546.1 \mathrm{~nm}$. Kirn [47] determined the refractive index at 15 wavelengths from 185.4 to $546.1 \mathrm{~nm}$ [45]. "The average deviation from those experimental points of Koch and Kirn lying in the visible region is $0.04 \%$ " [45]. Their formula is given by Eq. (19). 
McCarty et al. [40] (see pp. 1-31 therein) arrived at a formula similar to Eq. (19),

$$
r_{\infty}=0.99575-0.09069 \rho+1.227 \rho^{2},
$$

except that the last coefficient is 1.227 in [40] as opposed to 1.1227 in [45]. We believe this is a typographical error, given that in the same book [40] (pp. 1-15) they show the ClausiusMossotti equation

$$
\mathscr{R}_{\mathrm{CM}}=0.99575-0.09069 \rho+1.1227 \rho^{2},
$$

which is the same as Eq. (19).

Younglove [41] derived a density-, temperature-, and pressure-dependent Clausius-Mossotti relationship

$$
\mathscr{R}_{\mathrm{CM}}=A+B \rho+C \rho^{2}+D \rho^{3}+E T+F P,
$$

with densities in $\mathrm{mol} / \mathrm{dm}^{3}$ and the following coefficients: $A=0.20245443 \times 10^{-2}, \quad B=0.37171832 \times 10^{-6}, \quad C=$ $-0.92085013 \times 10^{-8}, D=-0.34065329 \times 10^{-11}, E=0$, and $F=0$ (see pp. 1-349 in [41]). Since in most formulas of specific refraction the density is in $\mathrm{g} / \mathrm{cm}^{3}$ instead of $\mathrm{mol} / \mathrm{dm}^{3}$ as in [41], Cauchy's coefficients for wavelength correction in Eq. (13) must be multiplied by the molar mass/1000. For $\mathrm{H}_{2}$, the molar weight is $2.01594 \mathrm{~g} / \mathrm{mol}$ [41].

\section{Temperature dependence of the refractive index}

Usually, the temperature dependence of the refractive index is not given explicitly, but rather implicitly through the temperature dependence of the density. We only found one explicit temperature-dependent formula of refractive index by McCarty [42] and many other explicit formulas for the temperature dependence of density (see, for example, [37,42]).

McCarty [42] used a modified Benedict-Webb-Rubin equation of state for para- $\mathrm{H}_{2}$ which considers the temperature dependence of density and expands specific refraction into a power series to get a formula for the temperature dependence of the refractive index (see p. 7 of [42])

$$
n=1.0509586594+0.091463402563 \epsilon^{0.41043983753} \text {. }
$$

This formula assumed that $T_{c}=32.93313976 \mathrm{~K}$, although not all digits are significant as McCarty gives $T_{c}=32.933 \mathrm{~K}$ elsewhere in the same work. A hint that not all digits are significant in Eq. (23) is the fact that the temperaturedependent density parameters are given, for example, as $A 1=$ $-1.0880215243 \pm 0.214$ (see p. 3 in [42]). This suggests that the error on $A 1$ propagated also to the refractive index and not all digits in Eq. (23) are significant. Equation (23) could also be corrected for wavelength with Eq. (14).

Jungwoon's review of $p-\mathrm{H}_{2}$ data gave the following corrections for temperature-dependent density [37]:

$$
\begin{aligned}
\rho_{\text {liquid }}= & \rho_{c}\left[1+1.7707(1-\epsilon)^{0.3817}\right], \\
\rho_{\text {gas }}= & \rho_{c}\{-0.01397+0.63032 \exp [-(1-\epsilon) / 0.16013] \\
& +0.37572 \exp [-(1-\epsilon) / 0.01365]\} .
\end{aligned}
$$

The corrections given by Eqs. (23) and (24) could be substituted into any or all of Eqs. (18), (19), and (22) above to get a more accurate estimate of the refractive index at each temperature. Subsequently, turbidity fitting using Eq. (8)
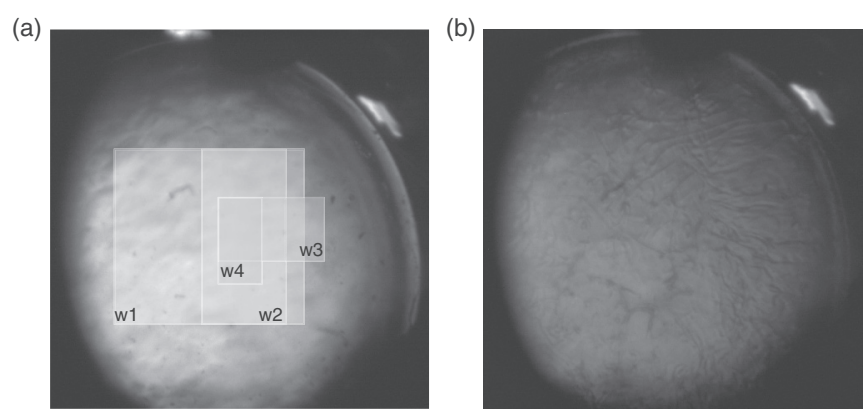

FIG. 4. Sample cell unit with $\mathrm{H}_{2}$ at (a) $T=35 \mathrm{~K}$ and (b) $T=$ 33.008 K. Four overlapping windows (marked w1-w4) were used for transmitted light intensity measurements. As the temperature approached $T_{c}=33.0065 \mathrm{~K}$, less light was transmitted (b).

could be done with refractive indices corresponding to actually measured temperature instead of using only $n_{c}$ for the entire temperature range. Additionally, correcting the specific refraction for both temperature with Eq. (23) and wavelength with Eq. (14) could improve the fitting of turbidity data.

\section{Fitting turbidity data for hydrogen}

In the following, we only show the fitting results for two refractive indices selected from Table III. A summary of some of our fitting results for $\mathrm{H}_{2}$ is shown in Table IV for a constant index of refraction, i.e., for $n_{c}=1.04911$ (measured at $546.2 \mathrm{~nm}$ ) as listed in the first row of Table III. Since the wavelength at which the refractive index was measured is given, we use Eq. (17) to correct the refractive index for the wavelengths of our particular filters. The procedure is similar for any other constant refractive index from Table III.

The second example uses one of the most complex refractive index formulas we found in the literature and includes the temperature dependence of the refractive index as shown in Eq. (23) (see p. 7 of [42]). The results for the temperaturedependent refractive index (23) are summarized in Table V. Given the complexity of the formula, we performed the following different fittings shown in Table $\mathrm{V}$ from Ref. [42]: (i) $n_{c}=1.0509586594=$ const; (ii) $n_{c}=1.0509586594=$ const, corrected with Eq. (17) for the wavelengths of our filters (since no wavelength was provided, we assumed that $\lambda \rightarrow \infty$, i.e., assumed that $n_{c}$ was derived from dielectric constant measurements); (iii) use the above filter-corrected values for $n_{c}$ and further add the temperature dependence from Eq. (23); and (iv) use the temperature dependence from Eq. (23) with no wavelength correction.

We fitted the turbidity data for each of the three colors (red, $669.4 \mathrm{~nm}$; green, $519.4 \mathrm{~nm}$; and blue, $465.2 \mathrm{~nm}$ ) and for each of the four window locations shown in Fig. 4(a). Representative turbidity plots versus reduced temperature data are shown in Fig. 5 for all three color filters used in this experiment. The data far from $T_{c}$ give the background turbidity $\tau \approx \tau_{B}$. The data closer to $T_{c}$, where $a$ is still small and $F(a)$ nearly constant (approximately equal to 8/3), give the isothermal compressibility $\tau \propto \kappa_{T} \propto \epsilon^{-\gamma}$ [slope approximately equal to $-\gamma$ in Fig. 5(a)]. Close to the critical point and for $a \geqslant 1, F(a)$ compensates the divergence of $\kappa_{T}$ and the turbidity exhibits a logarithmic behavior, thus allowing the determination of $\xi$ 
TABLE IV. Fitting results for a relatively small refractive index $n_{c}=1.04911$ measured at $546.2 \mathrm{~nm}$ [38].

\begin{tabular}{|c|c|c|c|c|c|c|}
\hline$n_{c}$ & Color & $\kappa_{T 0} \times 10^{9}\left(\mathrm{~Pa}^{-1}\right)$ & $\xi_{0}(\AA)$ & $\tau_{B}\left(\mathrm{~m}^{-1}\right)$ & $\chi^{2}$ & $R^{2}$ \\
\hline \multirow{12}{*}{$\begin{array}{l}1.04911 \\
(546.2 \mathrm{~nm})\end{array}$} & red & $76.2 \pm 4.3$ & $1.57 \pm 0.10$ & $0.99 \pm 0.14$ & 0.251 & 0.992 \\
\hline & red & $57.5 \pm 0$ & $1.12 \pm 0.03$ & $1.51 \pm 0.13$ & 0.447 & 0.986 \\
\hline & red & $85.2 \pm 1.4$ & $1.78 \pm 0$ & $0.81 \pm 0.12$ & 0.281 & 0.991 \\
\hline & red & $57.5 \pm 0$ & $1.78 \pm 0$ & $2.41 \pm 0.33$ & 3.674 & 0.887 \\
\hline & green & $68.8 \pm 5.5$ & $2.77 \pm 0.18$ & $0.83 \pm 0.19$ & 0.332 & 0.991 \\
\hline & green & $57.5 \pm 0$ & $1.20 \pm 0.10$ & $3.13 \pm 0.35$ & 3.44 & 0.905 \\
\hline & green & $41.7 \pm 1.1$ & $1.78 \pm 0$ & $1.63 \pm 0.22$ & 0.837 & 0.977 \\
\hline & green & $57.5 \pm 0$ & $1.78 \pm 0$ & $0 \pm 0.42$ & 6.143 & 0.831 \\
\hline & blue & $69.3 \pm 7.8$ & $3.36 \pm 0.28$ & $0.54 \pm 0.26$ & 0.594 & 0.986 \\
\hline & blue & $57.5 \pm 0$ & $2.92 \pm 0.05$ & $0.87 \pm 0.18$ & 0.637 & 0.985 \\
\hline & blue & $32.09 \pm 1.18$ & $1.78 \pm 0$ & $1.83 \pm 0.32$ & 1.813 & 0.957 \\
\hline & blue & $57.5 \pm 0$ & $1.78 \pm 0$ & $0 \pm 1.03$ & 36.428 & 0.129 \\
\hline \multirow{4}{*}{$\begin{array}{l}1.04857 \\
\text { from Eq. (17) }\end{array}$} & red & $78.0 \pm 4.4$ & $1.57 \pm 0.10$ & $0.99 \pm 0.14$ & 0.251 & 0.992 \\
\hline & red & $57.5 \pm 0$ & $1.09 \pm 0.03$ & $1.55 \pm 0.13$ & 0.483 & 0.985 \\
\hline & red & $87.21 \pm 1.42$ & $1.78 \pm 0$ & $0.81 \pm 0.12$ & 0.281 & 0.991 \\
\hline & red & $57.5 \pm 0$ & $1.78 \pm 0$ & $2.48 \pm 0.34$ & 4 & 0.877 \\
\hline \multirow{4}{*}{$\begin{array}{l}1.04929 \\
\text { from Eq. (17) }\end{array}$} & green & $68.3 \pm 5.5$ & $2.77 \pm 0.18$ & $0.83 \pm 0.19$ & 0.332 & 0.991 \\
\hline & green & $57.5 \pm 0$ & $2.41 \pm 0.04$ & $1.13 \pm 0.13$ & 0.382 & 0.99 \\
\hline & green & $41.4 \pm 1.1$ & $1.78 \pm 0$ & $1.63 \pm 0.22$ & 0.836 & 0.977 \\
\hline & green & $57.5 \pm 0$ & $1.78 \pm 0$ & $0 \pm 0.44$ & 6.488 & 0.821 \\
\hline \multirow{4}{*}{$\begin{array}{l}1.04977 \\
\text { from Eq. (17) }\end{array}$} & blue & $67.4 \pm 7.5$ & $3.35 \pm 0.28$ & $0.54 \pm 0.26$ & 0.594 & 0.986 \\
\hline & blue & $57.5 \pm 0$ & $2.98 \pm 0.05$ & $0.81 \pm 0.18$ & 0.62 & 0.985 \\
\hline & blue & $31.2 \pm 1.2$ & $1.78 \pm 0$ & $1.83 \pm 0.32$ & 1.811 & 0.957 \\
\hline & blue & $57.5 \pm 0$ & $1.78 \pm 0$ & $0 \pm 1.11$ & 41.736 & 0.002 \\
\hline
\end{tabular}

[slope approximately equal to $-v$ in Fig. 5(b)]. Note that a leveling off of the turbidity near $T_{c}$ can occur if the density is not strictly equal to the critical density. This question of density close to the critical value becomes very limiting when one approaches the critical temperature very closely [21]. This effect can be correctly analyzed only using the universal Isinglike parametric form of the fluid equation of state (see, for example, Refs. [48,49]). Such a complex analysis can be ignored for our present experimental approach of the critical point.
However, we note that when the density is off-critical, one observes a phase change at the coexistence temperature $T_{c x}$ instead of $T_{c}$. As a result, the temperature scale then becomes $T / T_{c x}-1$ instead of the reduced temperature $\epsilon=T / T_{c}-1$. Furthermore, the turbidity divergence is replaced by turbidity saturation. For a detailed explanation of the reasons behind the estimated slopes in Fig. 5, see the numerical estimation of the power law exponents based on the theoretical formula of turbidity given by Eq. (8) in Sec. IV E.
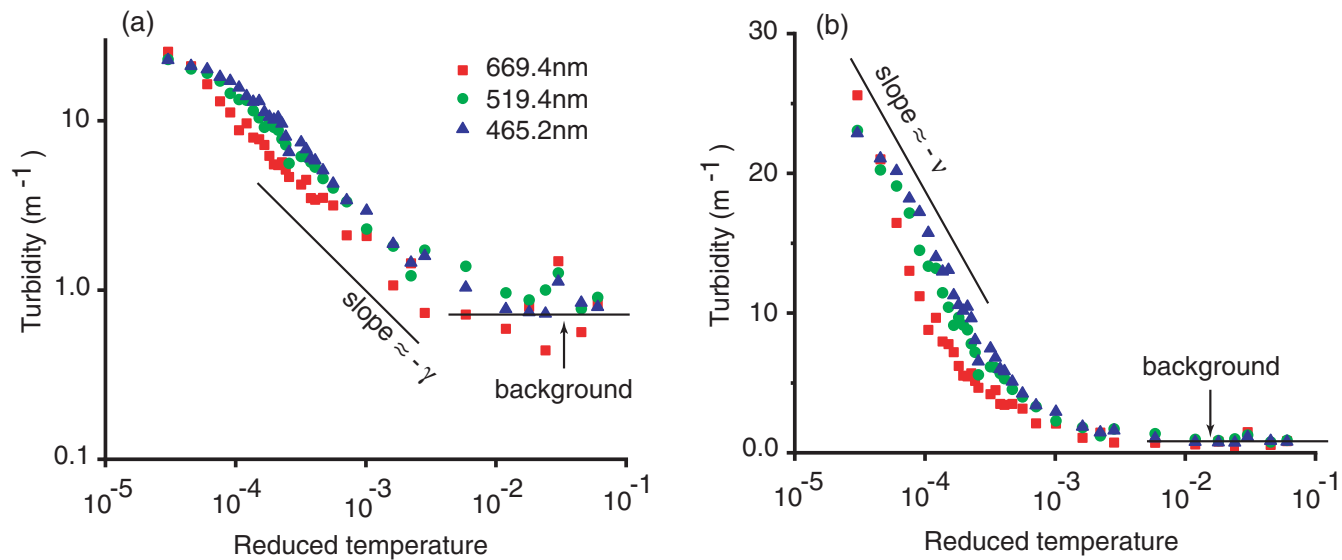

FIG. 5. Turbidity variation with reduced temperature in (a) $\log -\log$ and (b) semilogarithmic scale for all three filters. The region far from $T_{c}$ in (a) highlights the background contribution (horizontal line). Closer to $T_{c}$, the divergence of the isothermal compressibility leads to a straight fitting line with a slope approximately equal to $-\gamma$ in (a). The region very close to $T_{c}$ in the log-linear plot of (b) reveals the contribution of the interference term from which the correlation length can be determined [straight line with slope approximately equal to $2 v-\gamma \approx-v$ in (b)]. 
TABLE V. Fitting results for a temperature-dependent refractive index from Ref. [42]. The best fitting results are highlighted in bold for each index of refraction and filter color.

\begin{tabular}{|c|c|c|c|c|c|c|}
\hline$n_{c}$ & Color & $\kappa_{T 0} \times 10^{9}\left(\mathrm{~Pa}^{-1}\right)$ & $\xi_{0}(\AA)$ & $\tau_{B}\left(\mathrm{~m}^{-1}\right)$ & $\chi^{2}$ & $R^{2}$ \\
\hline \multirow{6}{*}{$\begin{array}{l}1.0509586594=\text { const } \\
\text { from Eq. (23) at } T_{c}\end{array}$} & red & $70.4 \pm 4.0$ & $1.56 \pm 0.10$ & $0.99 \pm 0.14$ & 0.251 & 0.992 \\
\hline & red & $57.5 \pm 0$ & $1.24 \pm 0.03$ & $1.36 \pm 0.11$ & 0.347 & 0.989 \\
\hline & green & $63.6 \pm 5.1$ & $2.76 \pm 0.18$ & $0.83 \pm 0.19$ & 0.332 & 0.991 \\
\hline & green & $57.5 \pm 0$ & $2.55 \pm 0.04$ & $1.00 \pm 0.13$ & 0.348 & 0.99 \\
\hline & blue & $64.1 \pm 7.2$ & $3.35 \pm 0.28$ & $0.54 \pm 0.26$ & 0.594 & 0.986 \\
\hline & blue & $57.5 \pm 0$ & $3.09 \pm 0.05$ & $0.72 \pm 0.18$ & 0.598 & 0.986 \\
\hline $1.05208=\mathrm{const}$ & red & $67.3 \pm 3.8$ & $1.56 \pm 0.10$ & $0.99 \pm 0.14$ & 0.251 & 0.992 \\
\hline from Eq. (17) at $T_{c}$ & red & $57.5 \pm 0$ & $1.31 \pm 0.03$ & $1.28 \pm 0.11$ & 0.305 & 0.99 \\
\hline $1.05284=\mathrm{const}$ & green & $58.9 \pm 4.7$ & $2.76 \pm 0.18$ & $0.83 \pm 0.19$ & 0.332 & 0.991 \\
\hline from Eq. (17) at $T_{c}$ & green & $57.5 \pm 0$ & $2.71 \pm 0.04$ & $0.87 \pm 0.13$ & 0.333 & 0.991 \\
\hline $1.05332=\mathrm{const}$ & blue & $58.2 \pm 6.5$ & $3.34 \pm 0.28$ & $0.54 \pm 0.26$ & 0.594 & 0.986 \\
\hline from Eq. (17) at $T_{c}$ & blue & $57.5 \pm 0$ & $3.31 \pm 0.05$ & $0.55 \pm 0.18$ & 0.579 & 0.986 \\
\hline 1.05208 & red & $59.6 \pm 3.1$ & $1.45 \pm 0.09$ & $0.94 \pm 0.14$ & 0.241 & 0.992 \\
\hline from Eq. (17) with Eq. (23) & red & $57.5 \pm 0$ & $1.39 \pm 0.03$ & $1.01 \pm 0.09$ & 0.237 & 0.993 \\
\hline 1.05284 & green & $49.4 \pm 3.7$ & $2.49 \pm 0.16$ & $0.78 \pm 0.19$ & 0.335 & 0.991 \\
\hline from Eq. (17) with Eq. (23) & green & $57.5 \pm 0$ & $2.82 \pm 0.04$ & $0.49 \pm 0.14$ & 0.382 & 0.99 \\
\hline 1.05332 & blue & $47.4 \pm 4.9$ & $2.98 \pm 0.24$ & $0.47 \pm 0.27$ & 0.601 & 0.986 \\
\hline from Eq. (17) with Eq. (23) & blue & $57.5 \pm 0$ & $3.44 \pm 0.06$ & $0.11 \pm 0.19$ & 0.656 & 0.985 \\
\hline \multirow{6}{*}{$\begin{array}{l}1.050958 \\
\text { with Eq. (23) }\end{array}$} & red & $62.2 \pm 3.3$ & $1.44 \pm 0.09$ & $0.94 \pm 0.14$ & 0.241 & 0.992 \\
\hline & red & $57.5 \pm 0$ & $1.32 \pm 0.03$ & $1.09 \pm 0.10$ & 0.251 & 0.992 \\
\hline & green & $53.0 \pm 4.0$ & $2.49 \pm 0.16$ & $0.78 \pm 0.19$ & 0.335 & 0.991 \\
\hline & green & $57.5 \pm 0$ & $2.66 \pm 0.04$ & $0.62 \pm 0.13$ & 0.35 & 0.99 \\
\hline & blue & $51.8 \pm 5.4$ & $2.97 \pm 0.24$ & $0.47 \pm 0.27$ & 0.602 & 0.986 \\
\hline & blue & $57.5 \pm 0$ & $3.21 \pm 0.05$ & $0.27 \pm 0.18$ & 0.608 & 0.986 \\
\hline
\end{tabular}

For each refractive index in Tables IV and V, the fitting results are shown as the mean plus or minus standard deviation (s.d.). The mean value was computed over the four fitting values corresponding to each of the four windows shown in Fig. 4. The fitting was performed in four different ways (see Tables IV and Fig. 6): (i) $\kappa_{T 0}, \xi_{0}$, and $\tau_{B}$ were all free parameters; (ii) $\kappa_{T 0}=57.5 \times 10^{-9} \mathrm{~Pa}^{-1}$ ( \pm 0 s.d.), while both $\xi_{0}$ and $\tau_{B}$ were free parameters; (iii) $\xi_{0}=1.78 \AA$ ( \pm 0 s.d.), while both $\kappa_{T 0}$ and $\tau_{B}$ were free parameters; and (iv) $\kappa_{T 0}=$ $57.55 \times 10^{-9} \mathrm{~Pa}^{-1}$ ( \pm 0 s.d. $), \xi_{0}=1.78 \AA$ ( \pm 0 s.d. $)$, and $\tau_{B}$ was a free parameter.

The reference values $\kappa_{T 0}=57.5 \times 10^{-9} \mathrm{~Pa}^{-1}$ and $\xi_{0}=$ $1.78 \AA$ are predicted using the master crossover functions [50] for one-component fluids constructed from the mean crossover functions for uniaxial three-dimensional Ising-like systems calculated in [27,50]. Consequently, when the four
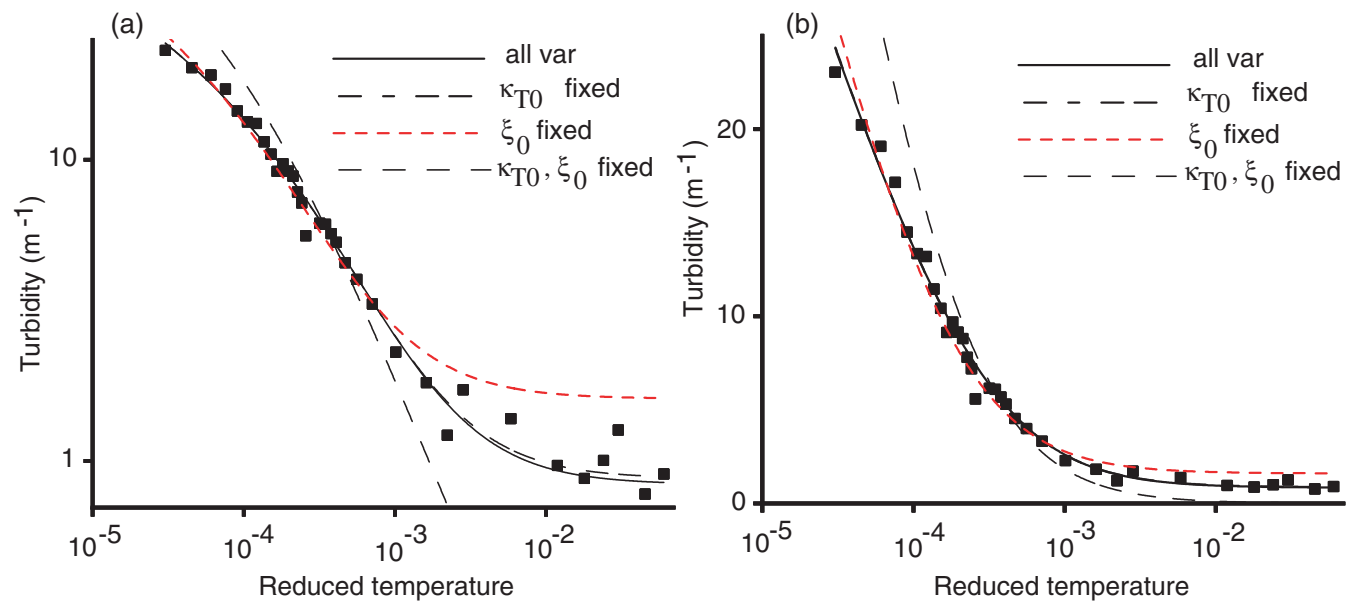

FIG. 6. (a) A log-log (a) and (b) a log-linear plot of experimental turbidity data (closed squares) and four different fitting conditions for a green filter with four windows with $\kappa_{T 0}, \xi_{0}$, and $\tau_{B}$ free parameters (solid line); $\kappa_{T 0}=57.5 \times 10^{-9} \mathrm{~Pa}^{-1}$, $\xi_{0}$, and $\tau_{B}$ free parameters (long-dash-short-dashed line); $\xi_{0}=1.78 \AA, \kappa_{T 0}$, and $\tau_{B}$ free parameters (short-dashed line); and $\kappa_{T 0}=57.5 \times 10^{-9} \mathrm{~Pa}^{-1}, \xi_{0}=1.78 \AA$, and $\tau_{B}$ free parameter (long-dashed line). 
critical coordinates of the critical point of any one-component fluid are known, the leading amplitudes of any singular physical property related to its corresponding master crossover function can be directly estimated [see, for example, Eqs. (71) and (72) and the data of Table III from [50] for the above referenced values of concern]. These values are very close to the $n-\mathrm{H}_{2}$ values $\kappa_{T 0}=57.56 \times 10^{-9} \mathrm{~Pa}^{-1}$ and $\xi_{0}=1.745 \AA$ as reported in [10], Table 1.6, p. 44. In the same table, the values for $p-\mathrm{H}_{2}$ were unknown for $\kappa_{T 0}$ and $\xi_{0}=1.54 \AA$ [10].

The fitting results in Table IV for each refractive index and filter color follow the above order of fitting parameter constraints. For example, the first row of Table IV is for $n_{c}=1.04911$ measured at $546.2 \mathrm{~nm}$ according to Ref. [38]. It contains a row for each of the three filters used in the experiment (red, green, and blue). For $n_{c}=1.04911$ measured at $546.2 \mathrm{~nm}$ for the red filter, there are four different fitting averages depending on which parameters were constant during the fitting procedure. The first row has all free parameters and reads $\kappa_{T 0}=(76.2 \pm 4.3) \times 10^{-9} \mathrm{~Pa}^{-1}, \quad \xi_{0}=(1.57 \pm$ $0.10) \AA$, and $\tau_{B}=(0.99 \pm 0.14) \mathrm{m}^{-1}$. The goodness of fit is measured by $\chi^{2}=0.251$ (the smaller the better) and adjusted $R^{2}=0.992$ (the larger the better).

The next row in Table IV has an index of refraction $n_{c}=1.04857$ from Eq. (17). This means that we used the experimentally measured index of refraction $n_{c}=1.04911$ (at $546.2 \mathrm{~nm}$ ) from the first row and Eq. (17) to correct for the corresponding wavelength of the filter used in our experiment (red, green, and blue).

Two of the four fitting averages for each case are obviously off due to very high $\chi^{2}$ and small, sometimes even negative $R^{2}$. This can be clearly seen also from Fig. 6, where we show only one example, i.e., the green filter with window 4 . The fitting with only $\xi_{0}=1.78 \AA$ fixed (short-dashed line) severely overestimates the background turbidity, whereas the fitting with both $\kappa_{T 0}=57.5 \times 10^{-9} \mathrm{~Pa}^{-1}$ and $\xi_{0}=1.78 \AA$ fixed (long-dashed line) does not even converge (see Fig. 6). The two fittings with either all three parameters $\kappa_{T 0}, \xi_{0}$, and $\tau_{B}$ free (solid line) or only $\kappa_{T 0}=57.5 \times 10^{-9} \mathrm{~Pa}^{-1}$ fixed (long-dashshort-dashed line) are almost indistinguishable from each other, except for some minute differences at large temperature visible only on a log-log plot [Fig. 6(a)]. Therefore, we did not further consider the fittings with $\xi_{0}=1.78 \AA$ fixed and the fittings with $\kappa_{T 0}=57.5 \times 10^{-9} \mathrm{~Pa}^{-1}$ and $\xi_{0}=1.78 \AA$ fixed. The only reasonable fittings are the first two lines of each filter color, i.e., with all three parameters free or with $\kappa_{T 0}=57.5 \times 10^{-9} \mathrm{~Pa}^{-1}$ fixed in our analysis.

Since we never used the fitting results with $\xi_{0}$ fixed, i.e., the third line in each fitting result shown in Table IV, and with both $\kappa_{T 0}$ and $\xi_{0}$ fixed, i.e., the fourth line in each fitting result shown in Table IV, such fitting results are no longer shown for the temperature-dependent refractive index example in Table $\mathrm{V}$. This was done in order to avoid showing an exceedingly large table of which we only use half of the fitting results for further analysis.

While Tables IV and V are comprehensive, we also presented the data in a condensed graphical form in Fig. 7. We notice that the three filters provided estimates that are clearly separated in nonoverlapping clusters. The red filter provides the closest estimate to the expected values of both

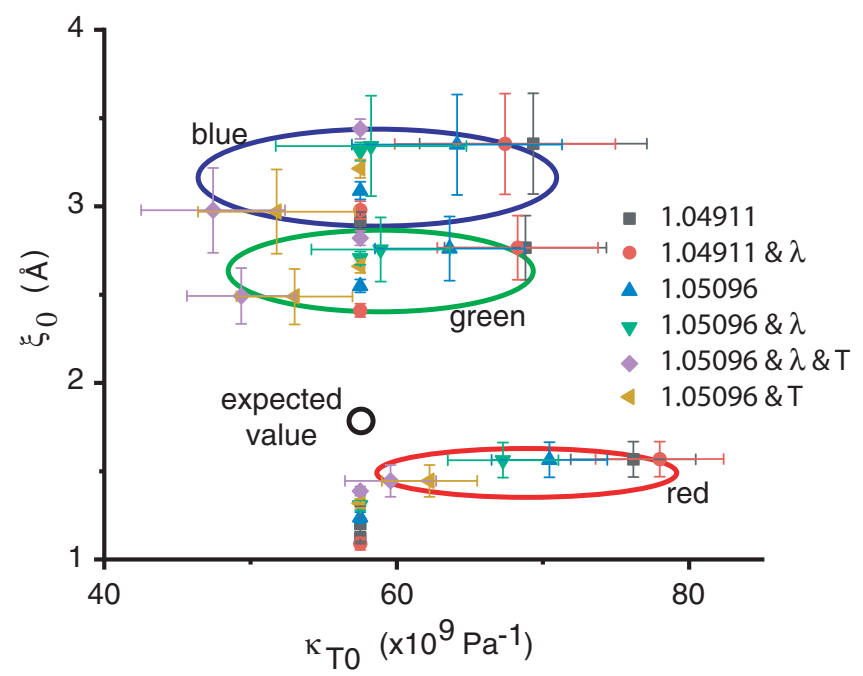

FIG. 7. Two representative refractive indices $n_{c}=1.04911$ and $n_{c}=1.05096$ with wavelength correction (marked by $\lambda$ ) and temperature-dependence (marked by T). There is a consistent increase of $\xi_{0}$ between red, green, and blue filters.

$\kappa_{T 0}$ and $\xi_{0}: \kappa_{T 0}=(59.6 \pm 3.1) 10^{-9} \mathrm{~Pa}^{-1}, \xi_{0}=(1.45 \pm$ $0.09) \AA$, and $\tau_{B}=(0.94 \pm 0.14) \mathrm{m}^{-1}$ for $n_{c}=1.050958$ corrected for both filter color with Eq. (17) and temperature with Eq. (23). It seems however that the filter color correction of the refractive index is less important than the temperature correction as the next best parameter estimation is $\kappa_{T 0}=$ $(62.2 \pm 3.3) \times 10^{-9} \mathrm{~Pa}^{-1}, \xi_{0}=(1.44 \pm 0.09) \AA$, and $\tau_{B}=$ $(0.94 \pm 0.14) \mathrm{m}^{-1}$.

For the green filter, $n_{c}=1.050958$ corrected for filter color gives $\kappa_{T 0}=(58.9 \pm 4.7) \times 10^{-9} \mathrm{~Pa}^{-1}, \xi_{0}=(2.76 \pm$ $0.18) \AA$, and $\tau_{B}=(0.83 \pm 0.19) \mathrm{m}^{-1}$. The next best fitting result is for $n_{c}=1.050958$ corrected for temperature with Eq. (23), which gives $\kappa_{T 0}=(53.0 \pm 4.0) \times 10^{-9} \mathrm{~Pa}^{-1}, \xi_{0}=$ $(2.49 \pm 0.16) \AA$, and $\tau_{B}=(0.78 \pm 0.19) \mathrm{m}^{-1}$.

For the blue filter, $n_{c}=1.050958$ corrected for filter color gives $\kappa_{T 0}=(58.2 \pm 6.5) \times 10^{-9} \mathrm{~Pa}^{-1}, \xi_{0}=(3.34 \pm$ $0.28) \AA$, and $\tau_{B}=0.54 \pm 0.26 \mathrm{~m}^{-1}$. The next best fitting result for this filter is for $n_{c}=1.050958$ corrected for temperature with Eq. (23), which gives $\kappa_{T 0}=(51.8 \pm 5.4) \times$ $10^{-9} \mathrm{~Pa}^{-1}, \xi_{0}=(2.97 \pm 0.24) \AA$, and $\tau_{B}=0.47 \pm 0.27 \mathrm{~m}^{-1}$.

With all free parameters, we notice from Fig. 7 that the estimation of $\kappa_{T 0}$ comes within one standard deviation of the expected value $\kappa_{T 0}=57.5 \times 10^{-9} \mathrm{~Pa}^{-1}$ in the case of $n_{c}=1.050958$. It is also clear from Tables IV and V that the estimated $\xi_{0}$ decreases with the filter's wavelength, while imposing a unique value of $\xi_{0}=1.78 \AA$ for all filters overestimates the $\kappa_{T 0}$ for the red filter and severely underestimates it for the green and blue filters. In addition, the estimated values of $\xi_{0}$ and $\tau_{B}$ are virtually the same whether all three parameters are free or if we impose $\kappa_{T 0}=57.5 \times 10^{-9} \mathrm{~Pa}^{-1}$. In light of the above results, the fitting method gives consistent results across all filters.

Equation (11) gives an explicit formula for the sensitivity of turbidity (8) to small changes in refractive index and wavelength. To complete the sensitivity analysis of turbidity (8), we also checked how the fitting results change when the critical temperature $T_{c}=33.0065 \mathrm{~K}$ is shifted a few $\mathrm{mK}$ around the 
(a)
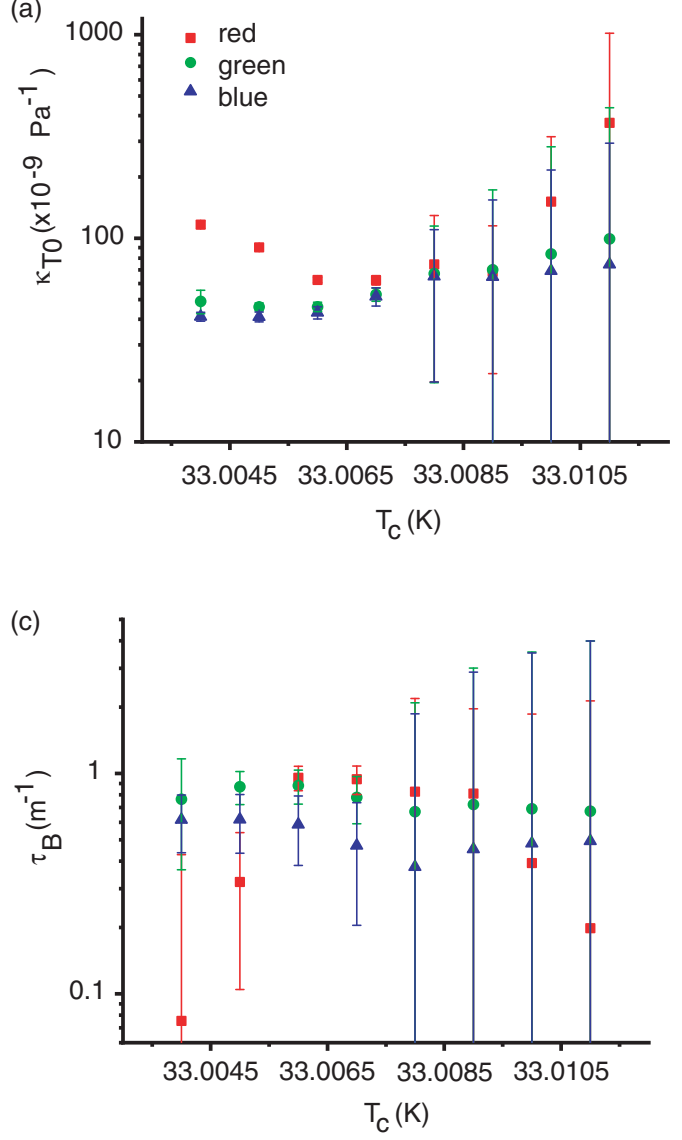

(b)

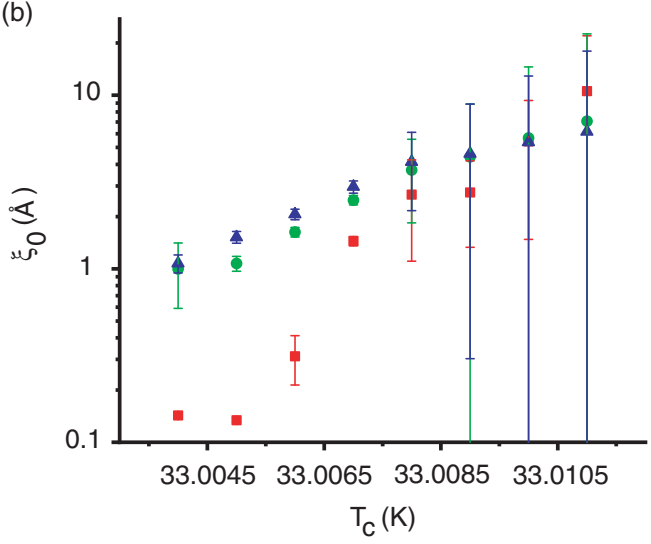

(d)

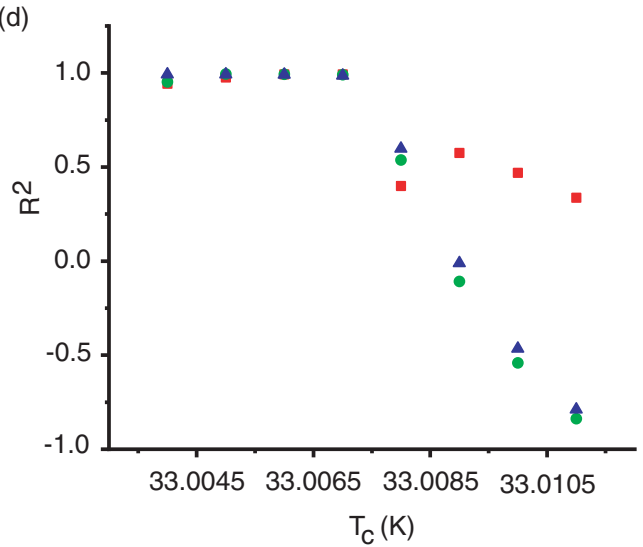

FIG. 8. Fitting parameters (a) $\kappa_{T 0}$, (b) $\xi_{0}$, and (c) $\tau_{B}$ all change slightly by changing the value of $T_{c}$ around the measured $33.0065 \mathrm{~K}$. (d) The best fitting with the smallest error bars and the largest adjuster $R^{2}$ is for $T_{c}=33.0065 \mathrm{~K}$.

measured value. Although we performed the analysis for all data, in Fig. 8 we only present the results for the last example from Table $\mathrm{V}$.

We notice from Fig. 8 that by slightly shifting $T_{c}$ below the measured value of $33.0065 \mathrm{~K}$ the error bars start rapidly increasing with the distance from $T_{c}=33.0065 \mathrm{~K}$. However, the adjusted $R^{2}$ [Fig. 8(d)] remains reasonably high, which indicates an acceptable fit. For temperatures slightly larger than $33.0065 \mathrm{~K}$, the fitting errors are very large and $R^{2}$ deteriorates quickly.

To conclude, for the index of refraction $n_{c}=1.050958$ that was corrected for filter wavelength with Cauchy's formula given by Eq. (14), we found that (i) $\kappa_{T 0}=(59.6 \pm 3.1) \times$ $10^{-9} \mathrm{~Pa}^{-1}$ and $\xi_{0}=(1.45 \pm 0.09) \AA$ for the red filter, (ii) $\kappa_{T 0}=(58.9 \pm 4.7) \times 10^{-9} \mathrm{~Pa}^{-1}$ and $\xi_{0}=(2.76 \pm 0.18) \AA$ for the green filter, and (iii) $\kappa_{T 0}=(58.2 \pm 6.5) \times 10^{-9} \mathrm{~Pa}^{-1}$ and $\xi_{0}=(3.34 \pm 0.28) \AA$ for the blue filter (see Table $\mathrm{V}$ for additional fitting results).

\section{Comparison with existing data}

One of the earliest experiments on liquid $\mathrm{H}_{2}$ was performed by Johnston et al. [51]. They measured the pressure versus volume for seven isotherms with temperature between 20.38 and $32.58 \mathrm{~K}$ of normal liquid $\mathrm{H}_{2}$. Even though the last isotherm at $32.58 \mathrm{~K}$ is not quite the critical temperature of $n-\mathrm{H}_{2}$ of $T_{c}=33.19 \mathrm{~K}$, it gives us a rough estimate of what should be the range of compressibility. We fitted their data (not shown) and found that the compressibility reaches almost $80 \times 10^{-9} \mathrm{~Pa}^{-1}$ at a pressure of $23.672 \mathrm{~atm}$ and temperature of $32.58 \mathrm{~K}$. The extrapolation to $p_{c}=12.89 \mathrm{~atm}$ (see Table I) gives a compressibility in the range (130-160) $\times 10^{-9} \mathrm{~Pa}^{-1}$. While isothermal compressibility diverges near $T_{c}$, these estimates give us a hint as to what to expect for the order of magnitude of the critical amplitude $\kappa_{T 0}$.

Theoretical predictions based on the van der Waals model have four different asymptotic forms (see Table III in [52]).

(i) For $\epsilon>0$ at $\rho_{c}$, the isothermal compressibility is given by $\kappa p_{c}=g \epsilon^{-\gamma}$, with $\gamma=1$ (as opposed to $\gamma=1.239$ from the three-dimensional Ising model) and $g=1 / 6$. As a result, the estimated critical amplitude is $\kappa_{T 0}=g / p_{c} \approx 127 \times$ $10^{-9} \mathrm{~Pa}^{-1}$.

(ii) For $\epsilon<0$ and along the $p-V$ coexistence curves, the isothermal compressibility is given by $\kappa p_{c}=g^{\prime}(-\epsilon)^{-\gamma^{\prime}}$, with $\gamma^{\prime}=1$ and $g^{\prime}=1 / 12$. As a result, the estimated critical amplitude is $\kappa_{T 0}^{\prime}=g^{\prime} / p_{c} \approx 63 \times 10^{-9} \mathrm{~Pa}^{-1}$.

(iii) For $\epsilon>0$ at $P_{c}$, the isothermal compressibility is given by $\kappa p_{c}=g_{p} \epsilon^{-\gamma}$, with $\gamma=2 / 3$ and $g_{p}=1 /\left(3^{1 / 3} \times 6\right)$. As a result, the estimated critical amplitude is $\kappa_{T 0} p=g_{p} / p_{c} \approx$ $88 \times 10^{-9} \mathrm{~Pa}^{-1}$.

(iv) For $\epsilon<0$ at $P_{c}$, the isothermal compressibility is given by $\kappa p_{c}=g_{p}^{\prime}(-\epsilon)^{-\gamma^{\prime}}$, with $\gamma^{\prime}=2 / 3$ and $g_{p}^{\prime}=1 /\left(3^{1 / 3} \times 6\right)$. As a result, the estimated critical amplitude is $\kappa_{T 0} p^{\prime}=$ $g_{p}^{\prime} / p_{c} \approx 88 \times 10^{-9} \mathrm{~Pa}^{-1}$. 
For a somewhat different theoretical perspective, Ivanov [53] summarized a large body of data and found a strong correlation between critical exponents and critical amplitudes. The isothermal compressibility is given by its usual formula [see Eq. (2) in [53]]

$$
p_{c} \kappa_{T}=\Gamma \epsilon^{-\gamma} .
$$

Based on Eq. (19), different critical point studies ranging from the three-dimensional Ising model to theoretical models of $\mathrm{CO}_{2}$ to experiments with $\mathrm{Xe}, \mathrm{NH}_{3}$, and $\mathrm{SF}_{6}$ concluded that the correlation between the amplitude $\Gamma$ and the critical exponent is [see Eq. (4) in [53]]

$$
\Gamma \gamma^{g}=\gamma_{0},
$$

with $g=7.15 \pm 0.16$ and $\gamma_{0}=0.23 \pm 0.01$. Based on [53], the critical amplitude $\kappa_{0}=\frac{\Gamma}{p_{c}}=\frac{\gamma_{0}}{\gamma^{g} p_{c}}=37.789 \times 10^{-9} \mathrm{~Pa}^{-1}$, where we used $\gamma=1.239$ and $p_{c}=1.315 \mathrm{MPa}$ (see Table I). Even if we assume that the values of $\gamma$ and $p_{c}$ are exact, the propagated relative error on $\kappa_{0}$ is $\frac{\delta \gamma_{0}}{\gamma_{0}}+\ln (\gamma) \delta g \approx 7.8 \%$, i.e., $\kappa_{0}=(37.8 \pm 2.9) \times 10^{-9} \mathrm{~Pa}^{-1}$. One of the main points of the study is that the critical amplitudes and critical coefficients vary widely from study to study even for the same substance. In addition, the study did not include any data for critical $\mathrm{H}_{2}$. Therefore, this estimation of critical amplitude $\kappa_{0}$ for $\mathrm{H}_{2}$ could be less than optimal.

To summarize, reasonable agreement was found with the expected compressibility $\left[\kappa_{T 0}=57.56 \times 10^{-9} \mathrm{~Pa}^{-1}\right.$ (see Table 1.6 on p. 44 in [10]) and $\kappa_{T 0}=57.5 \times 10^{-9} \mathrm{~Pa}^{-1}$ (in [50])], while a correlation length is found larger than the expected values of $\xi_{0}=1.745 \AA$ (see Table 1.6 on p. 44 in [10]) and $\xi_{0}=1.78 \AA$ (in [50]). The most likely reason is that the correlation length determination needs data very close to $T_{c}$ where even small off-criticality in density can cause large changes in turbidity.

\section{E. Estimates of power law exponents}

As we previously mentioned, the interference function $F(a)$ in Eq. (9), for a reasonable range of $1 \AA \leqslant \xi_{0} \leqslant 3 \AA$ and all wavelengths in the visible spectrum, is at least at $77.7 \%$ of its limit value of $8 / 3$ for all $\epsilon>10^{-3}$. In other words, for $\epsilon>$ $10^{-3}$ the interference function is quasiconstant and its effect on turbidity formula can be neglected. This approximation corresponds in $\mathrm{H}_{2}$ to an off-critical temperature of about $T-T_{c}>33 \mathrm{mK}$. In other words, for $T-T_{c}>33 \mathrm{mK}$ the temperature-dependent part of the turbidity formula given by Eq. (8) becomes

$$
\tau \approx A \pi T_{c}(1+\epsilon) \kappa_{0} \epsilon^{-\gamma}\left(1+a_{\chi} \epsilon^{\Delta}\right) 8 / 3 \propto \epsilon^{-\gamma},
$$

where the nonanalytical amplitude $a_{\chi}$ from Eq. (1) was used with $\Delta=0.5$. Since in this study the temperature range is $\epsilon \epsilon$ $\left(10^{-5}, 10^{-2}\right)$, no further corrections are needed. As a result, a plot of turbidity $\tau$ versus the reduced temperature $\epsilon$ should look like a power law with an exponent close to the value of $-\gamma$. However, the contribution of background turbidity $\tau_{B}$ becomes significant at large temperatures and may cover the above-mentioned power law trend. Therefore, there are two competing factors: (i) We need a wide range of large temperatures to get a good power law fit and (ii) as the temperature departs from $T_{c}$ towards larger temperatures the temperaturedependent turbidity $(1+\epsilon)\left(1+a_{\chi} \epsilon^{\Delta}\right) \epsilon^{-\gamma}$ becomes smaller than the background turbidity $\tau_{B}$. As a result, depending on the level of background turbidity, the temperature range where one can determine the critical exponent $\gamma$ is quite narrow. This may lead to significant errors in the estimation of $\gamma$ from the power law fit of turbidity $\tau$ versus reduced temperature $\epsilon$ for large temperatures.

At the other extreme, we are interested in finding the asymptotic behavior of the turbidity formula given by Eq. (8) for very small values of the reduced temperature $\epsilon$. Due to the asymptotic behavior of the correlation length $\xi=\xi_{0} \epsilon^{-v}$, the variable $a$ in the interference term $F(a)$ of Eq. (9) is asymptotically described by $a=2\left(k_{0} \xi\right)^{2} \propto \epsilon^{-2 v}$. As the reduced temperature decreases $(\epsilon \rightarrow 0)$, the variable $a$ diverges $(a \rightarrow \infty)$. While the second term of $F(a)$ vanishes as $a \rightarrow \infty$, the first term leads to an $\infty / \infty$ case, which can be solved with l'Hôpital's theorem and gives $\lim _{a \rightarrow \infty} F(a)=0$. After minor rearrangements of some terms, it can be shown that the power series approximation of $F(a)$ for the case when $a$ is very large, i.e., $\epsilon \rightarrow 0$, becomes

$$
\frac{2 \ln (2)}{a}+\frac{2 \ln (2)}{a^{2}}+O\left(a^{-3}\right) .
$$

Therefore, the asymptotic behavior of $F(a)$ as $a \rightarrow \infty$ is $F(a) \approx \frac{2 \ln (2)}{a} \propto \epsilon^{2 v}$. As a result, the temperature-dependent part of turbidity equation (8) becomes $\tau=A \pi T_{c}(1+$ $\epsilon) \kappa_{0} \epsilon^{-\gamma} \frac{2 \ln (2)}{2\left(k_{0} \xi_{0} \epsilon^{-v}\right)^{2}} \propto \epsilon^{2 \nu-\gamma}$.

The exponents $\gamma$ and $v$ are related by $\eta=2-\frac{\gamma}{v} \approx 0.02$ [54,55]. The Fisher exponent $\eta$, however, corresponds to an asymptotic correlation function different from OrnsteinZernike theory and Eq. (8) is no longer valid. Since our data are not close enough to the critical point in terms of density and temperature, we will not discuss this issue further.

The above detailed analysis is confirmed by the plot of the apparent power law exponent $\left(\tau \propto \epsilon^{x}\right)$ shown in Fig. 9(a), which was obtained from numerical simulation of the theoretical formula of turbidity given by Eq. (8). We notice that the absolute value of the apparent power law exponent $x$ for large temperatures is smaller than $\gamma$. As we notice from Fig. 9(a), it is impossible to establish in what temperature range the power law exponent is in any way related to $\gamma$ and/or $\nu$. To gain some insight into the effect of the critical exponents $\gamma$ and $v$ on the power law exponent of the theoretical turbidity formula given by Eq. (8), we carried out two sets of numerical simulations: with fixed $\gamma=1.239$ and variable $v \in\{0.5,0.6,0.7\}$ [see solid lines in Figs. 9(b)-9(d)] and with fixed $v=0.63$ and variable $\gamma \in\{1.1,1.2,1.3\}$ [see dotted lines in Figs. 9(b)9(d)]. We notice that critical exponent $v$ only changes the apparent power law exponent of turbidity versus reduced temperature for small temperatures [see Fig. 9(b)]. Indeed, all three curves for $v \in\{0.5,0.6,0.7\}$ converge quite rapidly to the same trace and are indistinguishable for $\epsilon \geqslant 0.01$ [see Fig. 9(c)]. At the same time, $\gamma$ covers a broad range of temperatures by shifting all power law exponents proportional to the value of $\gamma$. This trend is visible in all Figs. 9(b)-9(d) and the proportional shift covers $\epsilon \geqslant 5 \times 10^{-4}$.

To conclude, for reduced temperatures larger than $\epsilon \geqslant 5 \times$ $10^{-4}$, the apparent turbidity power law exponent is entirely 

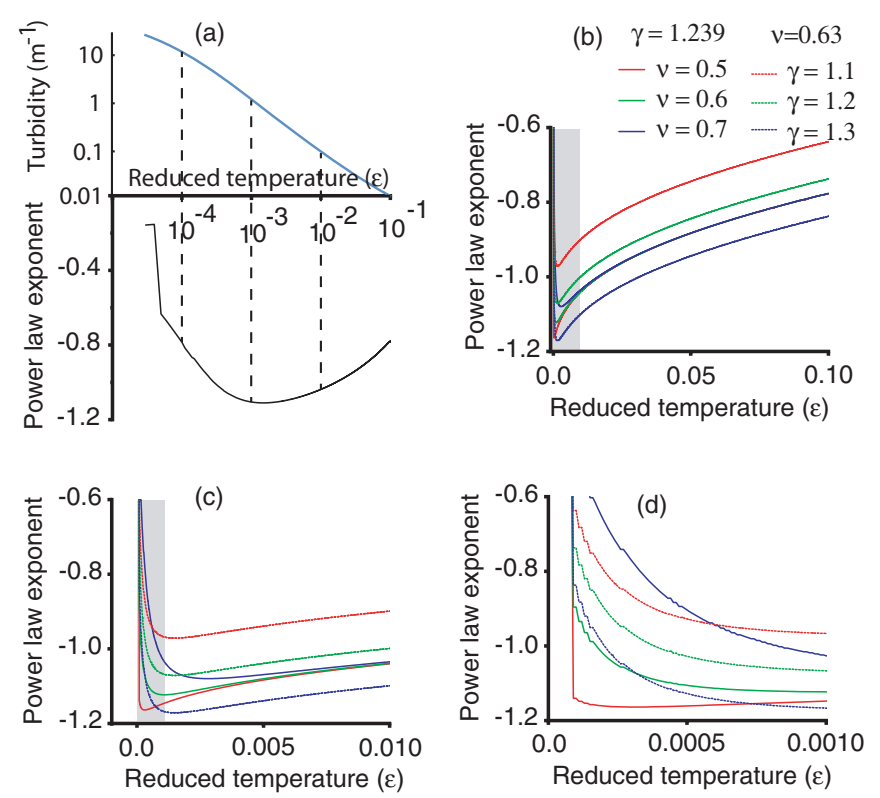

FIG. 9. Theoretical turbidity $\tau$ and its corresponding apparent power law exponent $x$ ( $\tau \propto \epsilon^{x}$ ). (a) The log-log plot of the theoretical turbidity formula given by Eq. (8) (top) and the corresponding apparent power law exponent $x$ (bottom). The critical exponents were $\gamma=1.239$ and $v=0.63$. The reduced temperature range in our experiment was $\epsilon \in\left(10^{-5}, 10^{-2}\right)$. (b) For a fixed $\gamma=1.239$ a slight change of $v$ only affects the power law exponent at low temperatures. For $\epsilon \leqslant 5 \times 10^{-3}$ and $v=0.5$ (red solid line), $v=0.6$ (green solid line), and $v=0.7$ (blue solid line), the power law exponent is very sensitive to $v$. This is highlighted by the magnification of the shaded area of (b), which is shown in (c), and by further magnifying the shaded are in (c), which is shown in (d). For fixed $v=0.63$, an increase in $\gamma$, i.e., $\gamma=1.1$ (red dotted line), $\gamma=1.2$ (green dotted line), and $\gamma=1.3$ (blue dotted line) in panels (b)-(d), shows that the power law exponent is most sensitive to $\gamma$ in the range $\epsilon \geqslant 5 \times 10^{-4}$.

determined by critical exponent $\gamma$. For reduced temperature $\epsilon \leqslant 5 \times 10^{-3}$, the critical exponent $v$ has a significant contribution to the apparent power law exponent.

\section{CONCLUSION}

Experiments near the critical point of cryogenic fluids like $\mathrm{H}_{2}$ require suppressing gravity effects and pose a series of challenges. First, for safety reasons, it is almost impossible to perform such experiments in microgravity. This is the reason why the only feasible alternative is the use of magnetic compensation to simulate weightlessness. Second, careful consideration must be given to the experimental conditions such that the $n-\mathrm{H}_{2}$ to $p-\mathrm{H}_{2}$ ratio is correctly determined. Thermophysical properties are sensitive to the $n-\mathrm{H}_{2}$ to $p-\mathrm{H}_{2}$ ratio. Given that the relaxation time constant for $\mathrm{H}_{2}$ is about $2500 \mathrm{~min}$ to reach the ortho-para equilibrium [34] and that experiments are performed within a couple of hours after filling in the cell, the sample cell contains mostly $n-\mathrm{H}_{2}$. Finally, since turbidity is measured through light transmission measurements, careful consideration was given to the light source and its intensity so that we minimize the effect of light-induced heating of the sample cell.
The paper's main results are concerned with (i) the careful design of HYLDE to generate good quality data very near $T_{c}$ through magnetic compensation of gravity, (ii) the use of image analysis for turbidity measurements corresponding to three different wavelengths centered on red, green, and blue filters, (iii) agreement of isothermal compressibility critical amplitude $\kappa_{T 0}$ with previous estimates, and (iv) better correlation length critical amplitude $\xi_{0}$ being obtained with data very near $T_{c}$ and density closer to critical value.

This study shows that light transmission measurements directly on the image of a critical fluid sample can give turbidity data with comparable precision as the traditional techniques using a laser beam. Using different wavelength filters gives more useful data and allows us to cross-check the critical amplitude values. This technique shows great promise as it allows both a detailed observation of the sample and local measurement of turbidity. When a density gradients exist, as is often the case in space or magnetic weightlessness experiments, different regions of interest of those images can be selected and compared. The more turbid regions correspond to fluid density closest to the critical density.

Concerning $\mathrm{H}_{2}$ under magnetic weightlessness, the data are fitted to the simplest formula (8) with isothermal compressibility, correlation length, and background turbidity as free parameters. One can see the extreme sensitivity of the fit to the refractive index value. Data the furthest from $T_{c}$ provide the background turbidity estimate and compressibility terms, while data close to $T_{c}$ give the correlation length. To summarize our results, for $n_{c}=1.050958$ corrected for the filter wavelength we found (i) $\kappa_{T 0}=(59.6 \pm 3.1) \times 10^{-9} \mathrm{~Pa}^{-1}$ and $\xi_{0}=(1.45 \pm 0.09) \AA$ for the red filter, (ii) $\kappa_{T 0}=(58.9 \pm$ $4.7) \times 10^{-9} \mathrm{~Pa}^{-1}$ and $\xi_{0}=(2.76 \pm 0.18) \AA$ for the green filter, and (iii) $\kappa_{T 0}=(58.2 \pm 6.5) \times 10^{-9} \mathrm{~Pa}^{-1}$ and $\xi_{0}=$ $(3.34 \pm 0.28) \AA$ for the blue filter (the uncertainty is one standard deviation in Table $\mathrm{V}$ ). These fitting results are in good agreement with previously published values for $n-\mathrm{H}_{2}$ susceptibility: $\kappa_{T 0}=57.56 \times 10^{-9} \mathrm{~Pa}^{-1}$ (see Table 1.6 on $\mathrm{p}$. 44 in [10]) and $\kappa_{T 0}=57.5 \times 10^{-9} \mathrm{~Pa}^{-1}$ (in [50]). In contrast, the correlation length was found larger than the expected value of $\xi_{0}=1.745 \AA$ (see Table 1.6 on p. 44 in [10]) and $\xi_{0}=1.78 \AA$ (in [50]). This discrepancy is due to the fact that data very near $T_{c}$ are affected by even minute density deviations from critical density.

A more accurate estimation of critical amplitudes could be achieved by controlling the density gradient throughout the sample to ensure that critical density is truly reached at some places. For that purpose, a dedicated sample could be placed either slightly off the axis at the compensation point height or on the axis of the compensation point.

The present experimental method, which uses image analysis at different wavelengths for a fluid under reduced gravity by magnetic means, offers several benefits to the study of critical point phenomena. First, magnetic compensation of gravity allows a very close approach to the critical temperature without gravity disturbances that usually affect critical fluids, e.g., buoyancy-driven instabilities and strong density gradients. Second, the light intensity can be made very weak to negligibly disturb the temperature of the sample, which is extremely important for cryogenic fluids. Third, each image covers the whole sample, which allows us to detect density 
inhomogeneities. In the case of imposed density gradients, the image analysis allows us to quantify the gradient. Fourth, other processes, such as phase transition, can be studied with the same setup. The critical temperature can thus be determined with high precision. Additionally, it is easy to vary turbidity intensities and cross-check results by using filters with different wavelengths.

\section{ACKNOWLEDGMENTS}

A.O. acknowledges partial support for this research through a grant from NASA South Carolina Space Grant Palmetto Academy. G.G., D.C., Y.G., and D.B. acknowledge partial support for this research from the Centre National d'Études Spatiales.
[1] T. Andrews, Philos. Trans. R. Soc. London 159, 575 (1869).

[2] J. Rowlinson, Nature (London) 244, 414 (1973).

[3] A. Lytle and D. Jacobs, J. Chem. Phys. 120, 5709 (2004).

[4] D. Beysens, in Materials Sciences in Space: A Contribution to the Scientific Basis of Space Processing, edited by B. Feuerbacher, H. Hamacher, and R. J. Naumann (Springer, Berlin, 1986), p. 191.

[5] D. Chandler and I. Oppenheim, J. Chem. Phys. 49, 2121 (1968).

[6] Phase Transitions and Critical Phenomena, edited by C. Domb and J. L. Lebowitz (Academic, New York, 2000), Vol. 18.

[7] A. Onuki, Phase Transition Dynamics (Cambridge University Press, Cambridge, 2002).

[8] V. Privman, P. Hohenberg, and A. Aharony, in Phase Transitions and Critical Phenomena, edited by C. Domb and J. L. Lebowitz (Academic, San Diego, 1991), Vol. 14.

[9] H. Stanley, Introduction to Phase Transitions and Critical Phenomena (Oxford University Press, Oxford, 1987).

[10] B. Zappoli, D. Beysens, and Y. Garrabos, Heat Transfers and Related Effects in Supercritical Fluids (Springer, Berlin, 2015).

[11] D. Chato, in Proceedings of the 45th AIAA Aerospace Sciences Meeting and Exhibit, Reno, 2007 (American Institute of Aeronautics and Astronautics, Reston, 2005), paper 2007-953, pp. 1-10.

[12] S. Mustafi, E. Canavan, W. Johnson, B. Kutter, and J. Shull, in Proceedings of the AIAA SPACE 2009 Conference and Exposition, Pasadena, 2009 (American Institute of Aeronautics and Astronautics, Reston, 2009), paper 2009-6584, pp. 1-10.

[13] C.-X. Wang, S.-H. Xu, Z.-W. Sun, and W.-R. Hu, Int. J. Heat Mass Transf. 53, 1801 (2010).

[14] D. Chatain, D. Beysens, K. Madet, V. Nikolayev, and A. Mailfert, Microgravity Sci. Technol. 18, 196 (2006).

[15] V. Nikolayev, D. Chatain, D. Beysens, and G. Pichavant, Microgravity Sci. Technol. 23, 113 (2011).

[16] R. Wunenburger, D. Chatain, Y. Garrabos, and D. Beysens, Phys. Rev. E 62, 469 (2000).

[17] L. Quettier, H. Felice, A. Mailfert, D. Chatain, and D. Beysens, Eur. Phys. J. Appl. Phys. 32, 167 (2005).

[18] D. Chatain and V. Nikolayev, Cryogenics 42, 253 (2002).

[19] V. G. Puglielli and N. C. Ford, Phys. Rev. Lett. 25, 143 (1970).

[20] C. Lecoutre, Y. Garrabos, E. Georgin, F. Palencia, and D. Beysens, Int. J. Thermophys. 30, 810 (2009).

[21] C. Lecoutre, R. Guillaument, S. Marre, D. Beysens, Y. Garrabos, and I. Hahn, Phys. Rev. E 91, 060101(R) (2015).

[22] L. S. Ornstein and F. Zernike, in 17th Proceedings of the Royal Netherlands Academy of Arts and Sciences (KNAW) (Elsevier, 1914), pp. 793-806.

[23] L. Ornstein and F. Zernike, Phys. Z. 19, 134 (1918).

[24] D. Beysens and Y. Garrabos, Physica A 281, 361 (2000).

[25] J. Zinn-Justin, Quantum Field Theory and Critical Phenomena (Clarendon, Oxford, 2002).
[26] C. Bagnuls and C. Bervillier, Phys. Rev. E 65, 066132 (2002).

[27] Y. Garrabos and C. Bervillier, Phys. Rev. E 74, 021113 (2006).

[28] Y. Garrabos, C. Lecoutre, S. Marre, R. Guillaument, D. Beysens, and I. Hahn, J. Stat. Phys. 158, 1379 (2015).

[29] D. T. Jacobs, S. M. Y. Lau, A. Mukherjee, and C. A. Williams, Int. J. Thermophys. 20, 877 (1999).

[30] A. Oprisan, J. J. Hegseth, G. M. Smith, C. Lecoutre, Y. Garrabos, and D. A. Beysens, Phys. Rev. E 84, 021202 (2011).

[31] G. Gandikota, D. Chatain, T. Lyubimova, and D. Beysens, Phys. Rev. E 89, 063003 (2014).

[32] T. Lyubimova, A. Ivantsov, Y. Garrabos, C. Lecoutre, G. Gandikota, and D. Beysens, Phys. Rev. E 95, 013105 (2017).

[33] C. Air Liquide and S. Division, Encyclopedie des Gaz (Elsevier, Amsterdam, 1976), p. 9.

[34] T. Charignon, P. Lloveras, D. Chatain, L. Truskinovsky, E. Vives, D. Beysens, and V. S. Nikolayev, Phys. Rev. E 91, 053007 (2015).

[35] H. Woolley, R. Scott, and F. Brickwedde, J. Res. Natl. Bur. Stand. 41, 379 (1948).

[36] H. M. Roder, G. E. Childs, R. D. McCarty, and P. E. Angerhofer, Survey of the Properties of the Hydrogen Isotopes below their Critical Temperatures, National Bureau of Standards (US) Technical Note 641 (US GPO, Washington, DC, 1973).

[37] C. Jungwoon, Liquid hydrogen properties, HANARO Report No. KAERI/TR-2723/2004, Korea Atomic Energy Research Institute, 2004 (unpublished).

[38] P. C. Souers, Cryogenic hydrogen data pertinent to magnetic fusion energy, Report No. UCRL-52628, Lawrence Livermore Laboratory, 1979 (unpublished).

[39] H. M. Roder, L. A. Weber, and R. D. Goodwin, Thermodynamic and Related Properties of Parahydrogen from the Triple Point to $100 \mathrm{~K}$ at Pressures to 340 Atmospheres, National Bureau of Standards (US) Monograph 94 (US GPO, Washington, DC, 1965).

[40] R. D. McCarty, J. Hord, and H. M. Roder, Selected Properties of Hydrogen, National Bureau of Standards (US) Monograph 168 (US GPO, Washington, DC, 1981).

[41] B. A. Younglove, J. Phys. Chem. Ref. Data 11, 1 (1982).

[42] R. D. McCarty, A modified Benedict-Webb-Rubin equation of state for parahydrogen, National Bureau of Standards Report No. NBSIR 74-357, 1974 (unpublished).

[43] H. Hoge and J. Lassiter, J. Res. Natl. Bur. Stand. 47, 75 (1951).

[44] J. Stewart, J. Chem. Phys. 40, 3297 (1964).

[45] R. J. Corruccini, Refractive Index and Dispersion of Liquid Hydrogen, NBS Technical Notes 323 (US GPO, Washington, DC, 1965). 
[46] J. Koch, Ark. Mat. Astron. Fys. 8 (20) (1912).

[47] M. Kirn, Ann. Phys. (Leipzig) 64, 566 (1921).

[48] Y. Garrabos, C. Lecoutre, S. Marre, and B. LeNeindre, J. Stat. Phys. 164, 575 (2016).

[49] Y. Garrabos, C. Lecoutre, S. Marre, B. LeNeindre, and I. Hahn, J. Stat. Phys. 165, 471 (2016).

[50] Y. Garrabos, C. Lecoutre, F. Palencia, B. Le Neindre, and C. Erkey, Phys. Rev. E 77, 021116 (2008).
[51] H. L. Johnston, W. E. Keller, and A. S. Friedman, J. Am. Chem. Soc. 76, 1482 (1954).

[52] D. C. Johnston, Advances in Thermodynamics of the van der Waals Fluid (Morgan and Claypool, San Rafael, 2014), pp. 2053-2571.

[53] D. Y. Ivanov, Dokl. Phys. 52, 380 (2007).

[54] M. E. Fisher, Rep. Prog. Phys. 30, 615 (1967).

[55] R. Kenna and B. Berche, Europhys. Lett. 105, 26005 (2014). 